\title{
An efficient method to derive statistical mechanical properties of concrete reinforced with spiral-shaped steel fibres in dynamic tension
}

\author{
Ying Wang* \\ Department of Civil and Environmental Engineering, University of Surrey, \\ Guildford, Surrey, GU2 7XH, United Kingdom \\ ying.wang@surrey.ac.uk (Corresponding Author)
}

\begin{abstract}
Yifei Hao
Tianjin University and Curtin University Joint Research Center of Structural Monitoring and Protection

Centre for Infrastructural Monitoring and Protection, School of Civil and Mechanical Engineering,

Curtin University

Bentley, WA, 6102, Australia. Yifei.Hao@ curtin.edu.au
\end{abstract}

\author{
Hong Hao \\ Tianjin University and Curtin University Joint Research Center of Structural Monitoring and \\ Protection \\ Centre for Infrastructural Monitoring and Protection, School of Civil and Mechanical Engineering, \\ Curtin University \\ Bentley, WA, 6102, Australia. Hong.Hao@ curtin.edu.au
}

\section{Xin Huang}

Department of Naval Architecture and Ocean Engineering, School of Civil Engineering, Tianjin University, 92 Weijin Road, Nankai District, Tianjin, 300072, P. R. China. huangxincn@tju.edu.cn

\begin{abstract}
Steel-fibre-reinforced concrete (SFRC) has been recognised as an effective solution to resist impact loading on structures. The reliable application and efficient design of SFRC structures depends on the knowledge of its mechanical properties. Since many important factors, including the locations and orientations of fibres and aggregates in concrete and the material properties of concrete matrix, are intrinsically random, the mechanical properties of SFRC present a high level of randomness. To accurately quantify them, effective statistical techniques are indispensable. Using traditional statistical techniques, a large quantity of data, from either experiments or numerical simulations, are needed to derive the correlation between the mechanical properties and the random factors. However, both ways are time-consuming and costly. Therefore, very little information regarding the statistical mechanical properties of SFRC can be found in the current literature. In this study, a kernel-based nonparametric statistical method is proposed to derive the statistical mechanical properties of SFRC with limited number of data. The behaviours of SFRC with randomly distributed spiral-shaped fibres and aggregates under impact loading are simulated using commercial software LS-DYNA. The simulation accuracy is validated by the experimental results. The influences of various volume fractions of fibres on dynamic increase factor (DIF) of the tensile strength of SFRC specimens under dynamic loadings at different strain rates are quantified through a prediction model obtained from kernel regression. The results demonstrate that the proposed method is able to estimate the DIF value of SFRC based on the tensile strength and strain rate, and to derive the statistical mechanical properties of SFRC.
\end{abstract}

\section{KEYWORDS}

Steel-fibre-reinforced concrete, spiral-shaped fibres, statistical mechanical properties, kernel density estimation, kernel regression, dynamic increase factor 


\section{INTRODUCTION}

Extreme loadings, including blast, hurricane, and earthquake, can lead to catastrophic results on the traditional reinforced concrete structures. Since concrete is intrinsically a brittle material with low tensile strength and strain capacities, increasing its tensile capacity can enhance the concrete structure safety. Adding fibres into concrete has been studied as an effective measure for decades. Fibres of different materials and various shapes have been proposed and their performance researched. Among them, steel-fibre-reinforced concrete (SFRC) has received increasing research attention, due to such advantages as the increased tensile strength [1], the improved toughness and the resistance against dynamic load [2], and reduced cracking [3].

The SFRC with spiral-shaped fibres has demonstrated to outperform SFRC with other types of steel fibres [4-10], due to its better bonds to concrete than other fibre types. Despite observations of good performance of concrete materials reinforced with spiral-shaped steel fibres, like concrete reinforced with other fibre types, fluctuations of mechanical properties of the SFRC materials have been observed in all the previous studies. This is because of the random distributions of fibres and aggregates in concrete matrix, fluctuations of material properties of each component in SFRC, and fluctuations in quality control of preparing the testing specimens, etc. Since these random fluctuations are inevitable in practice, to more reliably model the material performance it is better to derive the statistical material properties of SFRC.

To investigate the dynamic properties of SFRC, the current literature shows that there are two streams of methods, i.e., experimental tests and numerical simulations. Due to the high costs of experimental tests, only a limited number of high-speed impact tests were performed to obtain SFRC material properties corresponding to the relatively high strain rates. Thus, the dynamic increase factor (DIF) of SFRC as a function of strain rate for different SFRC is rarely available. Moreover, owing to the inevitable random fluctuations as discussed above, the limited testing data show random variations of obtained material properties. For example, 48 specimens were tested in an experimental study of the impact resistance capacities of SFRC and hybrid FRC subjected to drop weight impacts [11]. It showed that the coefficients of variations of the number of blows to cause the first crack and the total failure of the disc specimens were 0.59 and 0.52 , respectively, indicating significant variations of the SFRC capacities in resisting impact loads. In [12], the fibre distribution characteristics were found to depend on the direction of placing. Fibre distribution characteristics, including the degree of fibre dispersion, fibre unit number, and packing density, were found to strongly influence the ultimate flexural strength, while hardly affect the first cracking strength. In [8], extensive impact tests were conducted on SFRC specimens and a number of dynamic stress-strain curves under different strain rates obtained. The corresponding empirical DIF relations for spiral SFRC were proposed based on the testing data. Although those relations can be used to model dynamic strength increment with strain rate of SFRC, they cannot be unified and could be biased because they were derived from limited number of tests and the random fluctuations were not considered.

Computer modelling, or numerical testing, has been regarded as a reliable and cost effective approach to investigate the behaviour and properties of materials and structures under different loading conditions. To account for the randomly distributed coarse aggregates and steel fibres, modelling the SFRC specimens in mesoscale is needed. A number of researchers have performed mesoscale modelling of SFRC materials under static loading [13, 14], impact loading [7, 14, 15] and blast loading [16]. With distinctive consideration of steel fibres, coarse aggregates and mortar matrix, detailed observations such as stress and strain distributions, crack initiation and propagation, fibre-matrix interaction, can be made through these studies. However, as the distributions and orientations of discrete steel fibres and aggregates in SFRC mix are intrinsically random, which affect the simulated material properties, the mesoscale models adopted in the above studies can only represent the typical specimens with specific distributions and orientations of steel fibres and aggregates considered in the respective studies. To better understand the mechanical properties of SFRC materials with less biased results, statistical analysis accounting for random distributions of steel fibres and coarse aggregates are deemed necessary. To conduct statistical analysis and obtain 
unbiased results, traditional sampling techniques such as Monte Carlo simulations require as many, ideally infinite, samples as possible. This is very time consuming and not possible in practice.

To solve the above problems, efficient statistical analysis of the SFRC material properties accounting for the random distributions of steel fibres and aggregates is needed. Almost all the existing studies for efficient quantification of the statistical parameters of various problems use parametric statistics, which requires a predefined distribution, such as Weibull distribution, normal distribution, and log-normal distribution. Although Weibull distribution is used predominantly, its effectiveness was challenged against gamma or log-normal distribution function [17]. Alternatively, nonparametric statistics could be an efficient approach to derive the statistical properties of material properties. It is found that when the maximum likelihood point of kernel density estimation (KDE) stabilizes, the estimated distribution is usually close to the real distribution. This approach requires a much smaller number of samples than those using Monte Carlo method, therefore leading to significant savings in deriving the statistical parameters based on the number of testing and/or numerical data. To further reduce the computational efforts, a kernel regression method was developed and applied to derive the DIF variation according to the change of strain rate, based on limited number of test results [18]. In the latter study, reliable predication of DIF values under different strain rates, which are not available from tests or simulations, can be made. This preliminary study paved the way towards the establishment of an efficient statistical model for random materials.

In this paper, limited numerical simulations on the response of spiral-shaped SFRC in dynamic splitting tensile tests are performed using LS-DYNA. The accuracy of numerical model is verified by laboratory testing data. KDE is used to derive an unbiased statistical distribution of the dynamic tensile strength of spiral-shaped SFRC specimens at different strain rates with different ratios of fibres. Then, kernel regression is used to derive the prediction model of material properties to account for the random distributions of locations and orientations of aggregates and fibres in concrete based on the limited number of simulated data. A generalised model of DIF with respect to the strain rate and split tensile strength of SFRC is proposed to predict the dynamic strengths of SFRC under different strain rate.

\section{METHODOLOGY}

\section{Overview}

This study mainly involves the following six tasks:

1) Experimental tests of SFRC specimens with $1 \%$ steel fibre under impact are first conducted for validation purposes.

2) A commercial software LS-DYNA is used to simulate the behaviour of SFRC under dynamic splitting tension test in mesoscale considering coarse aggregates, spiral steel fibres and mortar matrix. The accuracy of the model predictions is verified by experimental test data. The validated model is then used to perform parametric studies on SFRC with different ratios of fibres and under different strain rates to provide data for the statistical analysis.

3) To provide a reliable distribution of dynamic strength of SFRC, KDE is used to analyse the static performance of SFRC with different ratios of fibres.

4) Kernel regression method is used to derive the predication models for tensile DIF of spiral SFRC material as a function of strain rate and static strength. Specifically, a 5-step method is proposed, which can achieve any prediction precision as requested. This prediction model is verified by the numerical simulation results.

5) $\mathrm{KDE}$ is used to derive the distribution of tensile DIF of SFRC at each strain rate.

6) Kernel regression method is used to derive the statistical model for the mechanical properties of SFRC.

KDE and kernel regression technologies used in this study are briefly summarised in the following. 


\section{Kernel Density Estimation}

$\mathrm{KDE}$ is an efficient tool to estimate distributions of random variables without prior knowledge, as detailed in [19]. The cumulative distribution function $F(x)$ of random variable $x$ can be estimated from $n$ random samples (observations) $\left\{X_{1}, \ldots, X_{n}\right\}$ as $\hat{F}(x)=n^{-1} \sum_{i=1}^{n} 1\left(X_{i} \leq x\right)$. Based on finite difference principle, the probability density function (PDF) can be estimated as:

$\hat{f}(x)=\frac{\hat{F}(x+h)-\hat{F}(x-h)}{2 h}=\frac{1}{2 n h} \sum_{i=1}^{n} 1\left(\left|X_{i}\right| \leq x+h\right)=\frac{1}{2 n h} \sum_{i=1}^{n} 1\left(\frac{\left|X_{i}-x\right|}{h} \leq 1\right)=\frac{1}{n h} \sum_{i=1}^{n} k\left(\frac{X_{i}-x}{h}\right)$

where $k(u)$ is a uniform density function on $[-1,1]: k(u)=\left\{\begin{array}{ll}\frac{1}{2}, & |u| \leq 1 \\ 0, & |u|>1\end{array} ; u=\frac{X_{i}-x}{h} ;\right.$ and $h$ is the bandwidth parameter. To generalize Eq. (1), $k(u)$ can be taken as any function that satisfies $\int_{-\infty}^{\infty} k(u) d u=1$, which is called as kernel function. The widely-used Gaussian kernel is:

$$
k(u)=\frac{1}{\sqrt{2 \pi}} e^{\frac{-u^{2}}{2}}-\infty \leq \mathrm{u} \leq \infty
$$

The estimation of distribution $\hat{F}(x)$ is therefore a summation of kernels. In this paper, the distributions of the DIF of SFRC under different strain rates are estimated by KDE.

\section{Kernel regression as nonparametric statistical method}

Generally, the concrete strengths and strain rates in the numerical experiments can be regarded as a vector of variables. The material properties can be generalised as a vector of the measured data. Their relationship can be expressed as:

$$
\mathbf{Y}=f(\mathbf{X})+\varepsilon
$$

where $\mathbf{Y}$ is a vector of the measured data, $\mathbf{X}$ is a vector/matrix of variables, $f(\mathbf{X})$ denotes the corresponding predications using model $f(\cdot)$, and $\varepsilon$ is the residue between the predicted and observed results.

In this study, we aim to derive a prediction model for SFRC material properties with respect to the concrete strengths and the strain rates of dynamic loading. Based on a previous study [18], the kernel regression method using local linear estimator has been demonstrated to be superior to other kernel regression methods. The relationship between measured data and the variables can be expressed as:

$$
f(\mathbf{X})=\frac{1}{n} \sum_{i=1}^{n} \frac{\left\{\hat{s}_{2}(\mathbf{X})-\hat{s}_{1}(\mathbf{X})\left(X_{i}-\mathbf{X}\right)\right\} K_{h}\left(X_{i}-\mathbf{X}\right) Y_{i}}{\hat{s}_{2}(\mathbf{X}) \hat{s}_{0}(\mathbf{X})-\left(\hat{s}_{2}(\mathbf{X})\right)^{2}}
$$

where $K_{h}\left(X_{i}-\mathbf{X}\right)=\frac{1}{h} K\left(\frac{X_{i}-\mathbf{X}}{h}\right)$ represents a kernel function with bandwidth $h ; \quad\left(X_{i}, Y_{i}\right)(i=1, \ldots, n)$ are the points with $n$ test data; and $\hat{s}_{r}(\mathbf{X})=\frac{1}{n} \sum_{i=1}^{n}\left(X_{i}-\mathbf{X}\right)^{r} K_{h}\left(X_{i}-\mathbf{X}\right)$. A Gaussian kernel is used in this study.

\section{Kernel regression method with multiple variables}

In [18], the process of the construction of statistical material property model for SFRC (DIF with respect to strain rate) were established, but the model has only one variable, therefore it can only account for the effects by one parameter, i.e., strain rate. It is understood that DIF of SFRC not necessarily depends on the strain rate only, but might also depend on other parameters such as fibre ratios and concrete strength. The extension to a multivariable kernel regression model has been conducted in the preliminary works in this study. However, the results are not satisfactory. Because the available testing data are very few, i.e., only 10 random strength values are available for each SFRC samples and only 3 ratios of steel fibres $(0.5 \%, 1 \%$, and $1.5 \%)$ are available. 
To improve the model and to apply this method to the prediction of the dynamic tension properties of SFRC, numerical simulations are carried out in this study and the simulated data are used to supplement the testing data in deriving the prediction and statistical models of SFRC with multiple variables. The following steps are proposed to investigate the effects of the static strength of concrete and the fibre ratios on DIF of SFRC:

1. Based on the limited data obtained from numerical simulations, the relationship between DIF and the strain rate under different concrete strengths and fibre ratios can be determined through kernel regression method, which is $\mathbf{Y}=f_{1}\left(x_{1}\right)$ under the fixed concrete strengths and fibre ratios. The coefficient of determination $\left(R^{2}\right)$ is used to demonstrate the superiority of the proposed method.

2. Based on the regression model obtained from kernel regression, more data on a number of specified strain rates (e.g., 2, 3, .., 18) are obtained through interpolation and extrapolation.

3. Using the data obtained in step 2, the relationship between DIF and concrete strength can be obtained through kernel regression method, which is $\mathbf{Y}=f_{2}\left(x_{2}\right)$ under the fixed strain rates and fibre ratios.

4. Based on the regression model obtained from kernel regression, more data on a number of specified concrete strengths are obtained through interpolation. Using the data obtained in this step and step 2, the relationship between DIF and strain rate/concrete strength can be plotted, which is intrinsically a prediction model, and is helpful in understanding the combined effects of these factors on the dynamic strength of SFRC.

5. The statistical mechanical properties of SFRC, including mean values and standard deviations, are derived based on prediction model.

6. The statistical model for the mean values and standard deviations are derived using kernel regression method.

It should be noted that the data obtained in Steps 2 and 3 can achieve any precision as requested, by changing the increment values for interpolation and extrapolation. This makes the prediction of material property based on limited data possible.

\section{RESULTS AND DISCUSSIONS}

\section{Laboratory tests for validation}

SFRC specimens with spiral fibres of $1 \%$ volume fraction were cast and tested under quasi-static loading using hydraulic testing machine and dynamic splitting tensile loading using split Hopkinson pressure bar (SHPB) systems. The dimension of specimens under dynamic splitting tension is $75 \mathrm{~mm}$ in diameter and $37.5 \mathrm{~mm}$ in height. Specifications of spiral fibres including yield strength, nominal length, wire diameter and coil pitch and diameter are summarised in Table 1. The quasi-static mechanical properties of SFRC with $1 \%$ spiral fibre are given in Table 2.

Table 1. Specifications of spiral steel fibres

\begin{tabular}{|c|c|c|c|c|c|}
\hline Yield strength & Wire diameter & $\begin{array}{c}\text { Nominal } \\
\text { length }\end{array}$ & $\begin{array}{c}\text { Nominal } \\
\text { aspect ratio }\end{array}$ & Coil pitch & Coil diameter \\
\hline $1300 \mathrm{MPa}$ & $0.56 \mathrm{~mm}$ & $15 \mathrm{~mm}$ & 27 & $10 \mathrm{~mm}$ & $5 \mathrm{~mm}$ \\
\hline
\end{tabular}

Table 2. Mechanical properties of SFRC under quasi-static loads

\begin{tabular}{|c|c|c|}
\hline Compressive strength & Tensile strength & Young's modulus \\
\hline $43.25 \mathrm{MPa}$ & $3.97 \mathrm{MPa}$ & $29.76 \mathrm{GPa}$ \\
\hline
\end{tabular}

Impact tests using Split Hopkison Pressure Bar (SHPB) testing system were carried out to study the dynamic splitting tensile behaviour of spiral SFRC at high strain rates. The schematic of SHPB system is given in Fig. 1. By releasing pressurised gas in the vessel, the striker bar is accelerated and impinges the incident bar to generate a one-dimensional compressive stress wave. Part of the wave is transmitted into the specimen sandwiched between the incident bar and transmitted bar while the rest 
is reflected. Having travelled forth and back a few times in the specimen to reach stress equilibrium, the stress wave propagates into the transmitted bar. The energy is then absorbed by the absorption bar and the buffer. Strain signals from gauges attached on the pressure bars (incident and transmitted) are amplified and received by data logger so that the incident, reflected and transmitted stress waves can be derived.

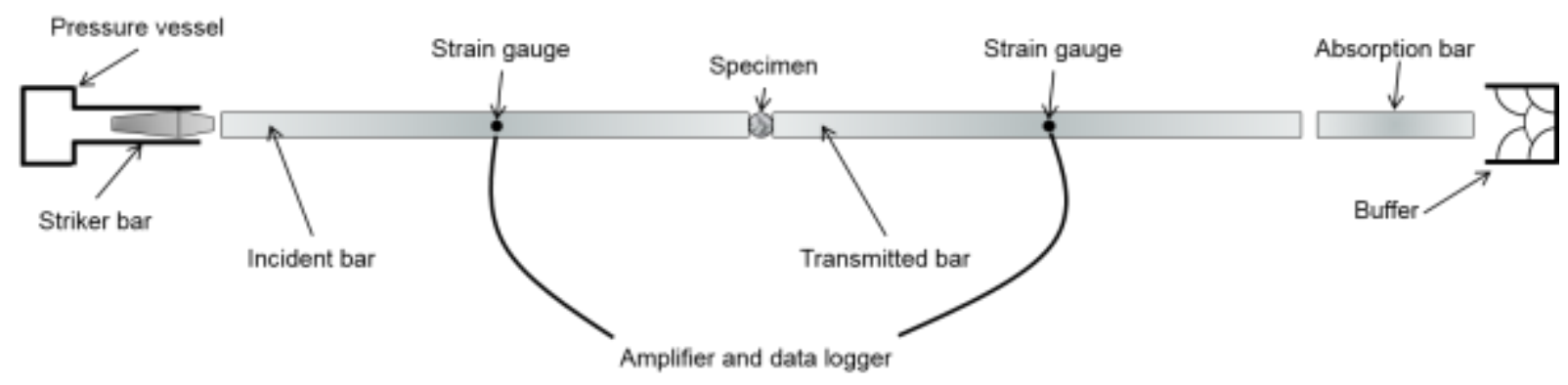

Figure 1. Schematic of SHPB testing system

In SHPB splitting tensile tests, as proposed by Tedesco et al. [20], the peak dynamic splitting stress $f_{t d}$ of the cylindrical specimen is proportional to the peak transmitted stress $\sigma_{\mathrm{T}}$, namely

$$
f_{t d}=\pi R^{2} \sigma_{T}
$$

in which $R$ is the radius of the pressure bars in SHPB.

Accordingly, following equations (Eqs. 6 and 7) can be used to estimate the loading rate $\dot{\sigma}$ and strain rate $\dot{\varepsilon}$ in the tested specimen.

$$
\begin{gathered}
\dot{\sigma}=\frac{f_{t d}}{\Delta t} \\
\dot{\varepsilon}=\frac{\dot{\sigma}}{E}
\end{gathered}
$$

where $\Delta \mathrm{t}$ is the time lag between the start and peak of the transmitted stress wave and $E$ is the Young's modulus of the material.

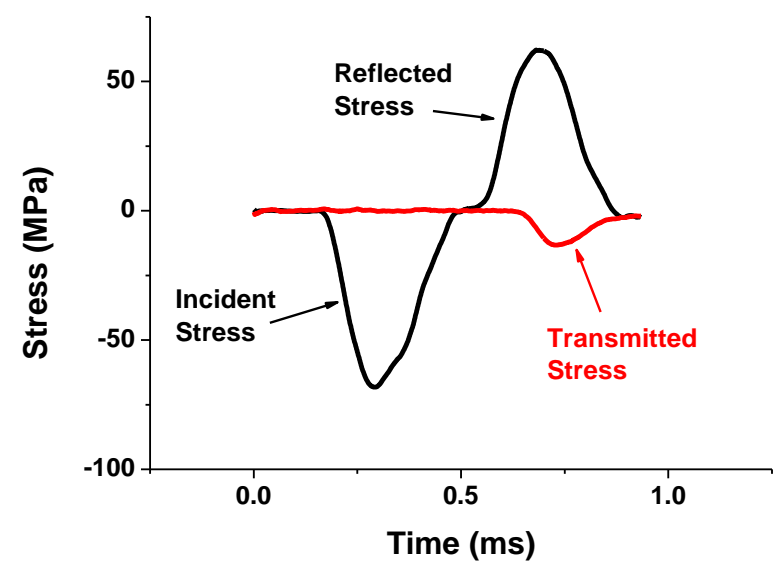

Figure 2. Typical histories of stress waves in SHPB tests 
Both the incident and transmitted bars used in the tests are identical in diameter of $75 \mathrm{~mm}$ and length of $2000 \mathrm{~mm}$. The striker bar, incident bar, transmitted bar and absorption bar are made of stainless steel with Young's modulus $200 \mathrm{GPa}$, density $7.8 \mathrm{t} / \mathrm{m}^{3}$, and Poisson's ratio 0.3. Typical stress histories recorded in SHPB splitting tensile test are given in Fig. 2, which are used to validate the numerical model in the following section.

\section{Numerical simulations}

Realistically the SFRC specimen should be modelled in 3D. However, a 3D mesoscale model of SFRC specimen requires a large number of elements because very fine mesh size is needed to model high-speed impact. This leads to a numerical model which requires large computer memory that may even exceed the capacity of the computer, and long simulation time. If relatively less refined mesh size is used, the simulation results are not necessarily accurate. To compromise the computational effort and simulation accuracy, in this study, the dynamic splitting tensile tests were simulated using LS-DYNA with shell elements of $0.5 \mathrm{~mm}$ thickness as a plane strain problem. In other words, the cylindrical specimen is modelled as a plane disc with the assumption that the performance in the longitudinal direction of the cylinder under split tension test is uniform. This is a reasonable assumption since it was found that the effect of fibre and aggregates in the longitudinal direction had less influence on the split tensile characteristics of cylindrical specimen [6]. Using this simplified model, sufficiently fine mesh can be used so that to minimize the modelling errors. In this study, the $75 \mathrm{~mm}$ diameter spiral SFRC specimens were modelled in mesoscale with consideration of randomly distributed spiral fibres, coarse aggregates and mortar matrix. The wire diameter, coil diameter, coil pitch and nominal length of the fibres are $0.56 \mathrm{~mm}, 5 \mathrm{~mm}, 10 \mathrm{~mm}$ and $15 \mathrm{~mm}$, respectively. The $2-\mathrm{m}$ long incident and transmitted pressure bars were also modelled by shell elements with thickness of 0.5 $\mathrm{mm}$. Instead of simulating the impact from striker bar, time-dependent nodal force was applied to the incident end of the incident bar as the input. These simplifications significantly reduce the modelling and simulation efforts. The accuracy of this simplified model in simulating split tension test of SFRC is verified first before it is applied to intensive numerical simulations.

The considered samples are SFRC cylinders with 1\%, 2\%, and 3\% spiral-shaped steel fibres. The random fibre locations and directions are generated by using MATLAB. Under each fibre ratio, 10 samples each subjected to 17 loading rates including quasi-static loading cases were simulated. In total 510 numerical simulations are carried out. Results from simulations considering quasi-static loading are used as reference strength for deriving DIF of SFRC materials.

It should be noted that one of the advantages of spiral fibres compared to other types of steel fibres was that they had better distribution in SFRC as was reported in [10]. In other words, with the same volume fraction, spiral fibres tend to be less likely to get agglomeration problem compared to other types of fibres. Therefore, SFRC with up to $3 \%$ steel fibres are studied in this paper.

Detailed information of the numerical simulation including mesoscale model, material models, mesh convergence, numerical model validation and parametric simulations is given below.

\section{Mesoscale model}

In the mesoscale model of spiral SFRC specimens, for simplification, coarse aggregates were assumed having circular shape with diameters in different ranges, representing the particle size distribution of coarse aggregates in concrete materials. It should be noted that it was proven in previous studies that modelling the irregular aggregate geometry by circular shape greatly simplifies the modelling effort and led to satisfactory predictions [6]. In this study, the aggregates are all modelled as circular. $10 \mathrm{~mm}$ was set as the maximum aggregate diameter, the same as the specimens used in laboratory tests. The coarse aggregates in mesoscale model were segmented into three ranges with diameters of $2-5 \mathrm{~mm}$, 5-8 $\mathrm{mm}$ and 8-10 mm, respectively. The modified Fuller's curve by Walraven [21] for 2D modelling was used to determine the aggregate particle size distribution as given below. 


$$
P\left(D<D_{0}\right)=P_{k}\left(1.065 D_{0}^{0.5} D_{\text {max }}^{-0.5}-0.053 D_{0}^{4} D_{\text {max }}^{-4}-0.012 D_{0}^{6} D_{\text {max }}^{-6}-0.0045 D_{0}^{8} D_{\text {max }}^{-8}-0.0025 D_{0}^{10} D_{\text {max }}^{-10}\right)
$$

in which $P$ denotes the cumulative percentage of coarse aggregates passing a sieve with aperture diameter $D, D_{\max }$ is $10 \mathrm{~mm}, P_{k}$ is the volumetric proportion of aggregates in the concrete mix, which is $40 \%$ in the present study.

The spiral fibres and coarse aggregates were generated with random positions and directions, with the avoidance of overlapping among fibres, aggregates and specimen boundaries [22]. An example of the developed SFRC specimen with 1\% spiral fibres is shown in Fig. 3.

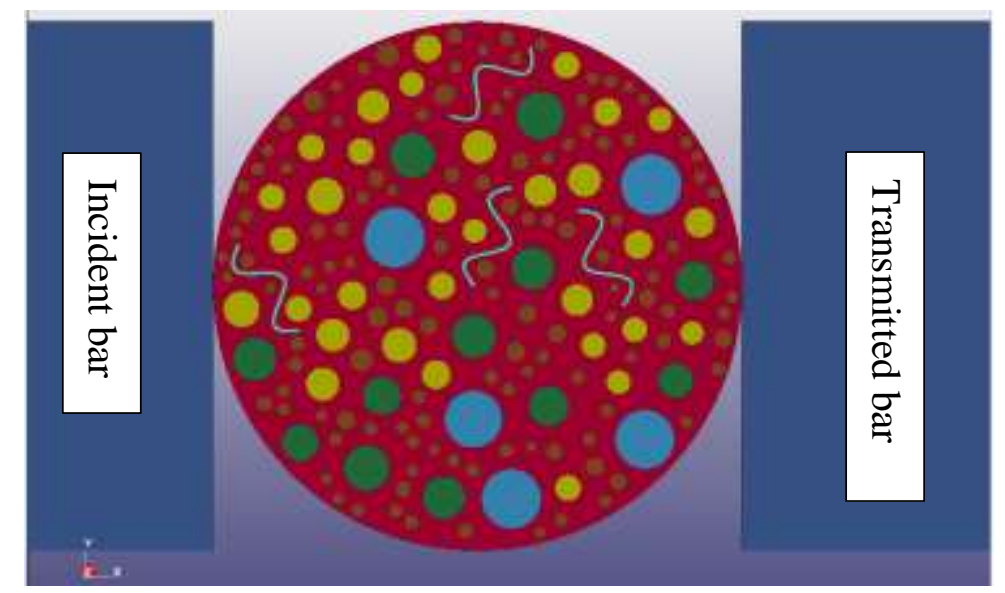

Figure 3. An example of developed SFRC specimen with 1\% spiral fibres in SHPB tests

The constituents in the SFRC specimen, i.e. mortar matrix, coarse aggregates and spiral fibres, were assumed to be perfectly bonded to each other. This assumption has been demonstrated by fibre pull-out tests where spiral fibres were fractured instead of being pulled out from the matrix because the spiral shape greatly increased the bonding between fibre and matrix [10]. Moreover, it should be noted that the interfacial transition zone (ITZ) was not considered in the meso-scale model because of the following reasons:

1) The typical thickness of ITZ is 10-50 micrometres. Considering ITZ with such thickness in the FE model extraordinarily increases the computational time and reduces the efficiency.

2) Apart from its thickness, other mechanical properties of ITZ are not well understood yet.

3) Most importantly, a previous study has compared the behaviour of mesoscale model with and without ITZ under dynamic loads and reported that ITZ had only insignificant influence on DIF [23]. As this study focuses on deriving DIF model of SFRC, ITZ was not considered in the simulation.

\section{Material models}

Release III of the K\&C plasticity model is adopted to model mortar matrix in the simulation. It is a three-invariant model using three shear-failure surfaces with the consideration of damage and strain rate effects. Based solely on the unconfined compressive strength, this model in LS-DYNA is able to automatically generate other modal parameters. The strain rate effect on the compressive and tensile strength is defined by introducing the DIF. In the simulation the compressive DIF (CDIF) relations for mortar matrix are adopted from [23] while the tensile DIF (TDIF) relations are adopted from [24]. They are given in Eqs. 9 and 10, respectively.

$$
\begin{aligned}
& C D I F=0.0419(\log \dot{\varepsilon})+1.2165 \text { for } \dot{\varepsilon} \leq 30 s^{-1} \\
& C I D F=0.8988(\log \dot{\varepsilon})^{2}-2.8255(\log \dot{\varepsilon})+3.4907 \quad \text { for } \quad 30 s^{-1} \leq \dot{\varepsilon} \leq 1000 s^{-1} \\
& T D I F=0.26(\log \dot{\varepsilon})+2.06 \quad \text { for } \dot{\varepsilon} \leq 1 s^{-1}
\end{aligned}
$$




$$
\begin{array}{r}
T D I F=2(\log \dot{\varepsilon})+2.06 \text { for } 1<\dot{\varepsilon} \leq 2 s^{-1} \\
T D I F=1.4433(\log \dot{\varepsilon})+2.2276 \quad \text { for } 2 s^{-1}<\dot{\varepsilon} \leq 150 s^{-1}
\end{array}
$$

Coarse aggregates are modelled by Pseudo Tensor model. The DIFs of coarse aggregates in the present study are adopted from [25] as below (Eqs. 11 and 12).

$$
\begin{gathered}
C D I F=0.0187(\log \dot{\varepsilon})+1.2919 \text { for } 1 s^{-1} \leq \dot{\varepsilon} \leq 220 s^{-1} \\
C I D F=1.8547(\log \dot{\varepsilon})^{2}-7.0914(\log \dot{\varepsilon})+9.6674 \quad \text { for } \quad 220 s^{-1} \leq \dot{\varepsilon} \leq 1000 s^{-1} \\
T D I F=0.0598(\log \dot{\varepsilon})+1.3588 \text { for } \quad 10^{-6} s^{-1} \dot{\varepsilon} \leq 0.1 s^{-1} \\
T I D F=0.5605(\log \dot{\varepsilon})^{2}+1.3871(\log \dot{\varepsilon})+2.1256 \quad \text { for } \quad \dot{\varepsilon}>0.1 s^{-1}
\end{gathered}
$$

The steel fibres are modelled by Piecewise Linear Plasticity material model from the LS-DYNA database and the pressure bars are modelled by the Isotropic Elastic Material model as they remain elastic in SHPB tests. The parameters of the materials are summarised in Table 3.

Table 3. Material parameters in numerical simulations

\begin{tabular}{llll} 
Material & Model in LS-DYNA & Input parameter & Value \\
\hline Mortar matrix & MAT_72R3 & Density & $2200 \mathrm{~kg} / \mathrm{m}^{3}$ \\
& & Unconfined compressive strength & $35 \mathrm{MPa}$ \\
& & Poisson's ratio & 0.18 \\
\hline Coarse aggregate & MAT_16 & Density & $2750 \mathrm{~kg} / \mathrm{m}^{3}$ \\
& & Unconfined compressive strength & $160 \mathrm{MPa}$ \\
& & Poisson's ratio & 0.20 \\
\hline Steel fibre & MAT_24 & Density & $7800 \mathrm{~kg} / \mathrm{m}^{3}$ \\
& & Young's modulus & $200 \mathrm{GPa}$ \\
& & Poisson's ratio & 0.3 \\
\hline Pressure bar & MAT_1 & Yield stress & $1300 \mathrm{MPa}$ \\
& & Density & $7800 \mathrm{~kg} / \mathrm{m}^{3}$ \\
& & Young's modulus & $200 \mathrm{GPa}$ \\
& & Poisson's ratio & 0.3
\end{tabular}

\section{Mesh convergence and model validation}

Mesh sensitivity tests were carried out to determine the optimal mesh size that yields accurate numerical simulation results with efficient use of computational time. In the mesh sensitivity tests mesh sizes of $0.56 \mathrm{~mm}, 0.28 \mathrm{~mm}$ and $0.14 \mathrm{~mm}$ were considered and simulation results, namely transmitted stress and crack opening velocity, were compared. Comparisons of the simulation results with different mesh sizes are given in Figs. $4 \mathrm{a}$ and $4 \mathrm{~b}$. As can be seen, results from numerical simulation using mesh size of $0.28 \mathrm{~mm}$ match well with those from simulation using $0.14 \mathrm{~mm}$ mesh size, whereas simulation considering $0.56 \mathrm{~mm}$ mesh size yields apparently different prediction of transmitted stress and velocity of splitting crack opening. Therefore mesh size of $0.28 \mathrm{~mm}$ is used in the subsequent models for accurate and efficient computational simulations. 


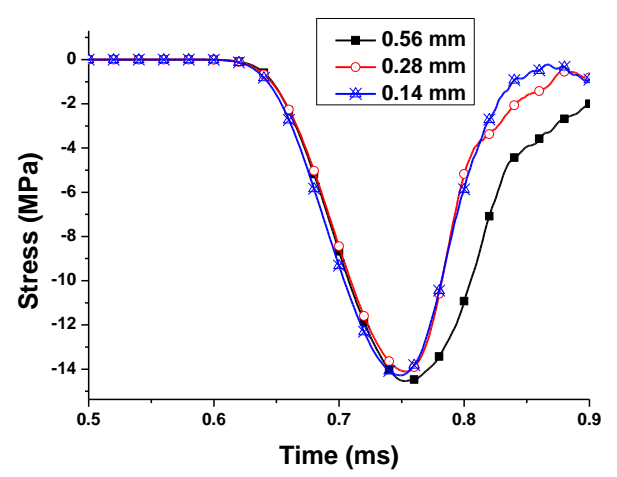

(a) Transmitted stress

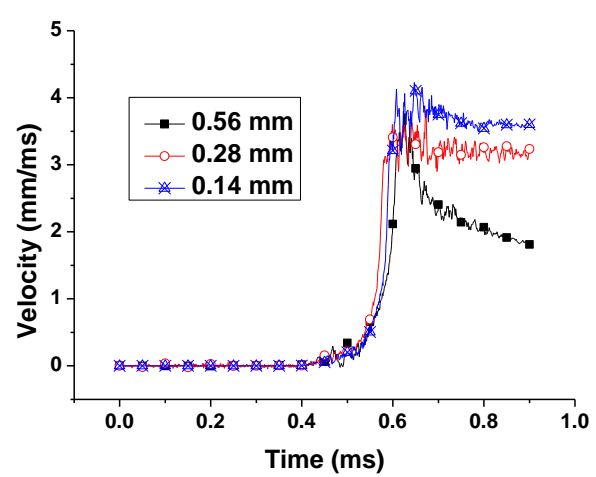

(b) Crack opening velocity

Figure 4. Comparison of transmitted stresses and crack opening velocities from simulations using different mesh sizes

To validate the numerical model, the model is used to simulate a dynamic split tension test reported in [9]. In this test, SFRC specimen with $1 \%$ spiral fibres was diametrically placed in between the pressure bars and loaded by an incident stress wave as given in Fig. 5. The same incident stress wave was used as the input in numerical simulation, and the reflected and transmitted stress waves were recorded and compared to those obtained from the test. It can be observed in Fig. 5 that the numerical simulation yields satisfactory results as compared to the test data, demonstrating the reliability of the numerical model, especially the accuracy in predicting the peak transmitted stress as it is the indicator of the splitting tensile strength of the SFRC specimen in SHPB splitting tensile tests.

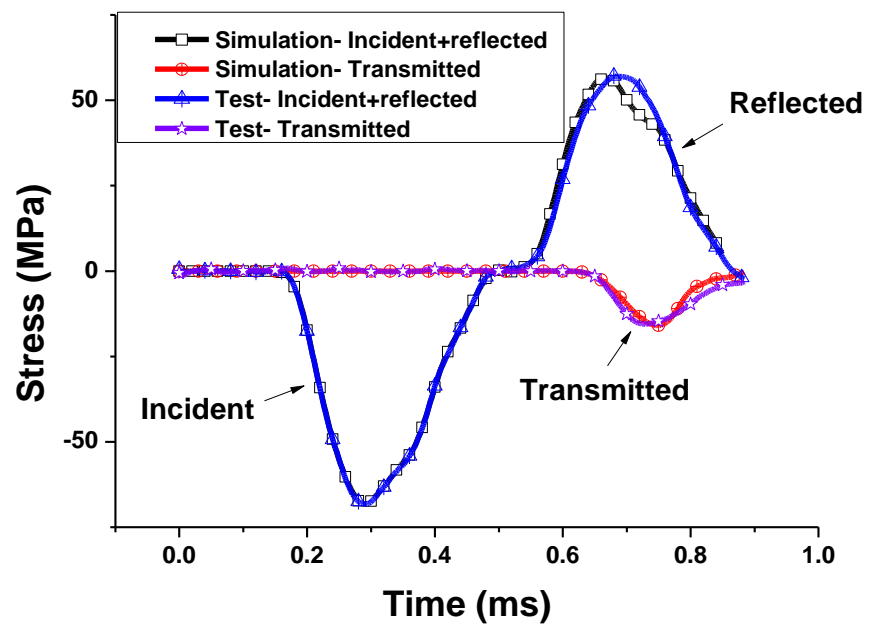

Figure 5. Comparison of histories of stress waves from numerical simulation and test

\section{Parametric simulations}

For statistical analysis of the mechanical properties of spiral SFRC, in particular the tensile DIF in the present study, database from numerical simulation results needs to be built. For spiral SFRC specimens containing $1 \%, 2 \%$ and $3 \%$ spiral fibres, 10 specimens were modelled for each fibre volume fraction case with randomly distributed spiral fibres and coarse aggregates. Fig. 6 gives examples of spiral SFRC specimens with $1 \%, 2 \%$ and $3 \%$ fibre fractions. For each specimen, simulation under quasi-static loading was firstly conducted for obtaining the quasi-static properties as the reference to determine DIF. Dynamic splitting tensile tests using SHPB were then simulated with 
16 loading rates, namely 16 strain rates ranging from $1 \mathrm{~s}^{-1}$ to $20 \mathrm{~s}^{-1}$. In total 510 simulations were conducted.
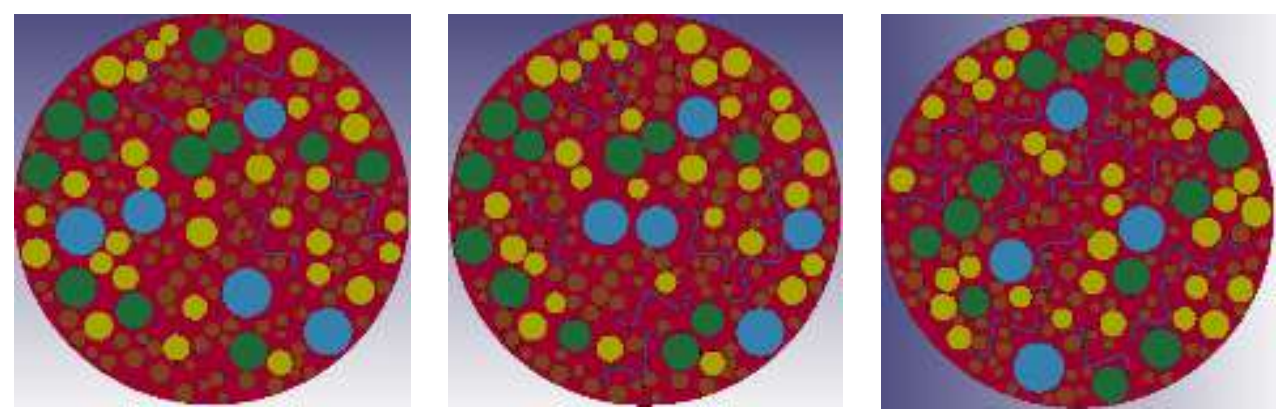

Figure 6. Examples of spiral SFRC specimens with 1\%, 2\% and 3\% fibre fractions

The obtained DIFs of different spiral SFRC specimens with different fibre volume fractions under different strain rates are plotted in Fig. 7. As can be observed, with the same fibre ratio but different distributions and orientations of spiral fibres and aggregates in the SFRC specimen, the DIF values vary. It can also be observed in Fig. 7 that the DIFs of SFRC specimens with $1 \%$ spiral fibres do not show significant scattering. The most significant scattered DIFs are observed for those with $2 \%$ spiral fibres, and the DIFs from SFRC specimens containing 3\% spiral fibres are less scattered than those with $2 \%$ spiral fibres. The above observations might be because that when the fibre volume fraction is small, namely $1 \%$ in the present study, the influence from steel fibres on DIFs is quite limited. When the fibre fraction is increased to $2 \%$ in the SFRC composite, the distributions and orientations of fibres play an important role in contributing to the dynamic splitting tensile strength, which results in more significantly scattered DIFs as shown in Fig. 7b. When the fibre ratio is further increased to $3 \%$, as can be seen in Fig. 6, spiral fibres tend to be more evenly distributed in SFRC specimens, thus the variation in DIFs tend to be less intensive than those of $2 \%$ spiral SFRC specimens. From Fig. 7, it can also be seen that increasing the number of spiral fibres in SFRC specimen does not necessarily always increase the DIF, indicating the significant influence of fibre distribution and orientation in SFRC specimens on dynamic material properties. In the three fibre volume cases considered in the study, the level of DIF variation increases with the strain rate. This could be attributed to the way of damage propagation in the specimen. Under quasi-static and low strain rate loading, SFRC specimen damage initiates at weak locations such as the interface between mortar matrix and aggregates or mortar matrix and steel fibres, and propagates along the weak sections. Increasing the loading and strain rate, damage propagates quickly and has less time to find the weak sections. For example, it was observed in many previous experimental tests and numerical simulations that the damage of concrete specimens under quasi-static loading usually occurs along the mortar-aggregate interface, whereas the damage occurs throughout the entire specimen with intensive aggregate damage when the loading rates are high [26]. Because all components of SFRC specimen are susceptible to damage when subjected to high-rate loading, and different components (mortar, aggregates and steel fibre) have different strengths, the dynamic SFRC strength is therefore more affected by the distributions of aggregates and steel fibres, which results in more significant variations in DIF with increasing strain rate. The results in Fig. 7 indicate the importance of statistical analysis of SFRC materials with the consideration of random aggregates and fibre distributions. 


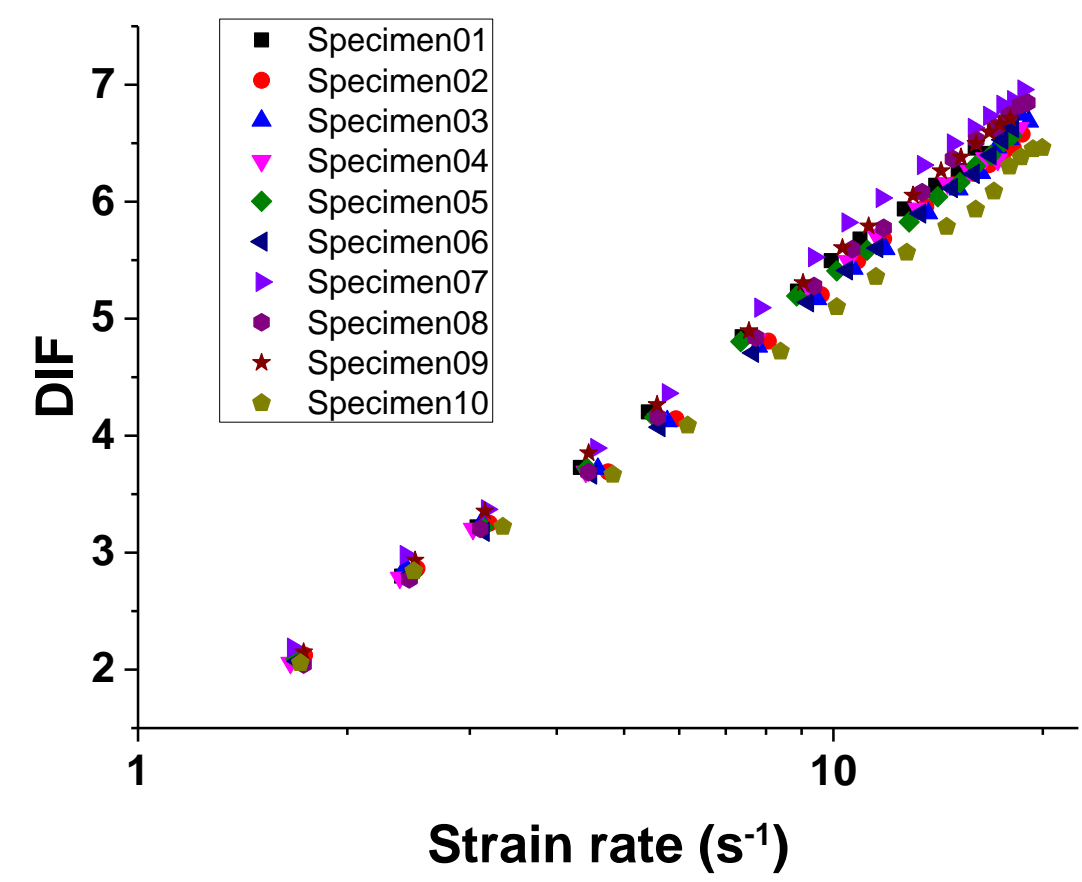

a) DIFs of $1 \%$ spiral SFRC specimens

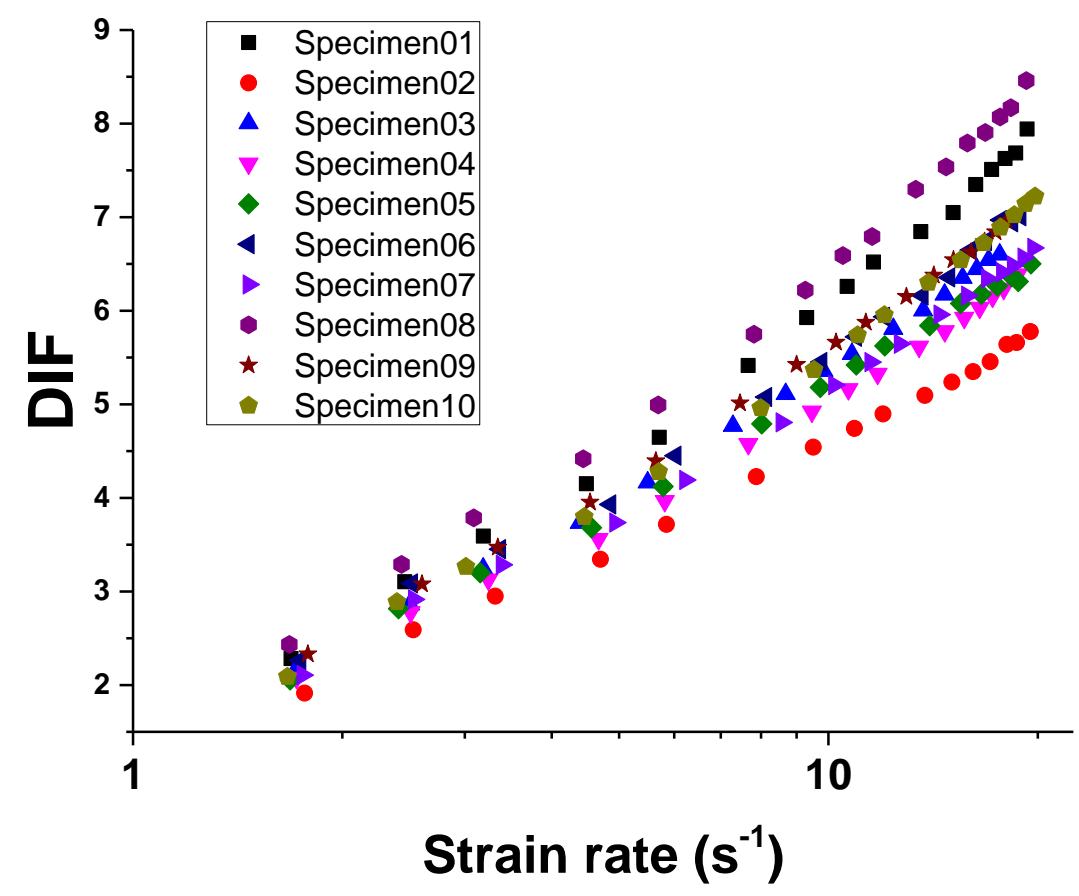

b) DIFs of $2 \%$ spiral SFRC specimens 


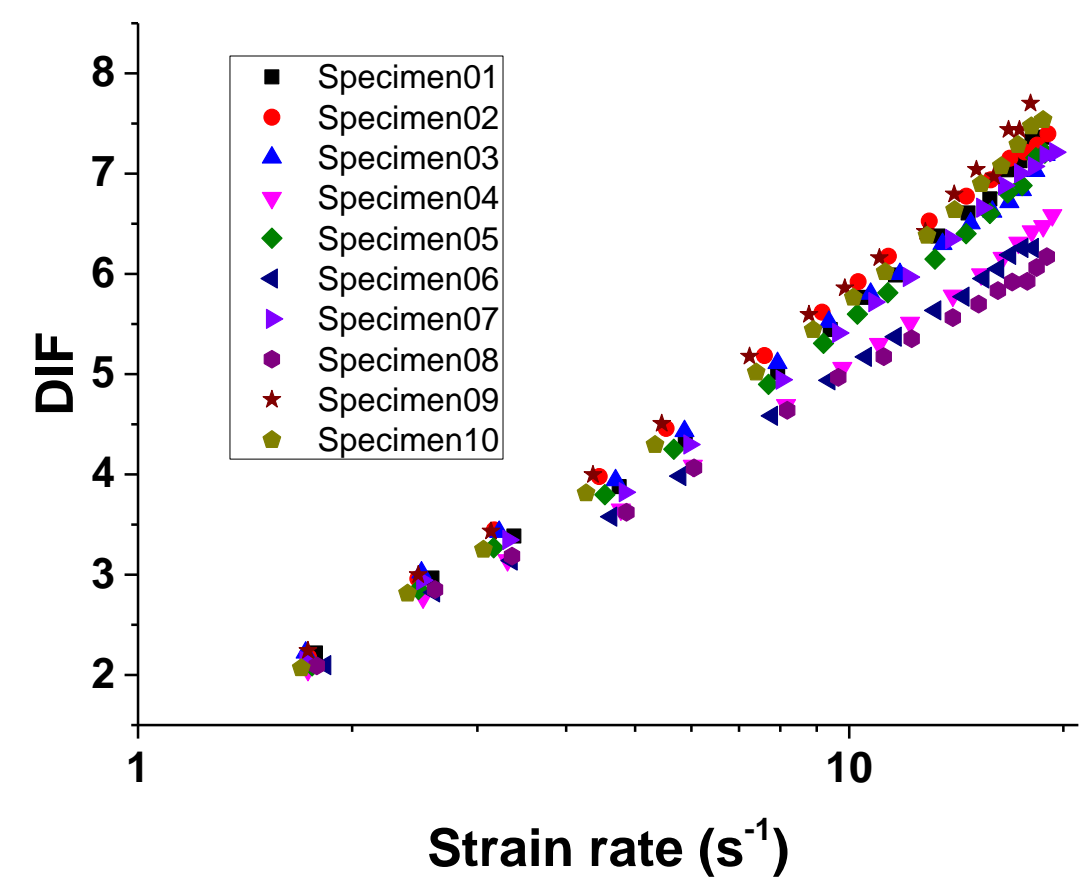

c) DIFs of $3 \%$ spiral SFRC specimens

Figure 7. Summary of DIFs of SFRC specimens with $1 \%, 2 \%$ and $3 \%$ fibre fractions

It should also be noted that the above numerical results are obtained with the randomly distributed fibres in the matrix. In reality, depending on the construction quality control fibre agglomeration might occur so that fibres are not necessarily evenly distributed in the concerete matrix. However, a previous study demonstrated that spiral-shaped fibres tend to remain at locations they were placed because their spiral shapes make them difficult to move during pouring and vibrations [10]. Therefore, they are more evenly distributed than other fibre types. Fibre agglomeration, which is undesirable in mixing fibres in SFRC and is very much dependent on the construction quality control, lead to biased fibre distributions in matrix is not considered in the present study.

\section{KDE for static strength distribution estimation}

In this paper, the static strengths of 40 samples with random aggregate and fibre orientations and locations are simulated for each scenario (SFRC with 1\%,2\% and 3\% fibre fractions). The results are used to find the stability point of the maximum likelihood points of the distributions estimated by KDE. As can be seen from Fig. 8 and Table 4, if only 5 data is used, unstable estimation is expected; however, estimation results from 10 data are already close to those from 40 data (with less than $1.5 \%$ difference for all three scenarios). Although the maximum likelihood points stabilize only when 30 data are used, 10 data can provide a very close estimation. To save expensive computational costs, only data from 10 samples are used in the rest of the derivation.

Table 4. Maximum likelihood points for the drift of the second story

\begin{tabular}{|c|c|c|c|}
\hline Number of data & 1\% spiral SFRC & 2\% spiral SFRC & 3\% spiral SFRC \\
\hline 5 & $3.331(-3.81 \%)$ & $3.177(-4.05 \%)$ & $3.37(-1.55 \%)$ \\
\hline 10 & $3.472(0.26 \%)$ & $3.274(-1.12 \%)$ & $3.377(-1.34 \%)$ \\
\hline 20 & $3.461(0.06 \%)$ & $3.263(-1.45 \%)$ & $3.359(-1.87 \%)$ \\
\hline 30 & $3.424(-1.13 \%)$ & $3.327(0.48 \%)$ & $3.41(-0.38 \%)$ \\
\hline 40 & 3.463 & 3.311 & 3.423 \\
\hline
\end{tabular}


It should be noted that four significant digits are kept in the results in Table 4 and those in the following tables 5-7, based on the setting in MATLAB. This setting, i.e. the number of significant digits, can be easily changed when necessary.

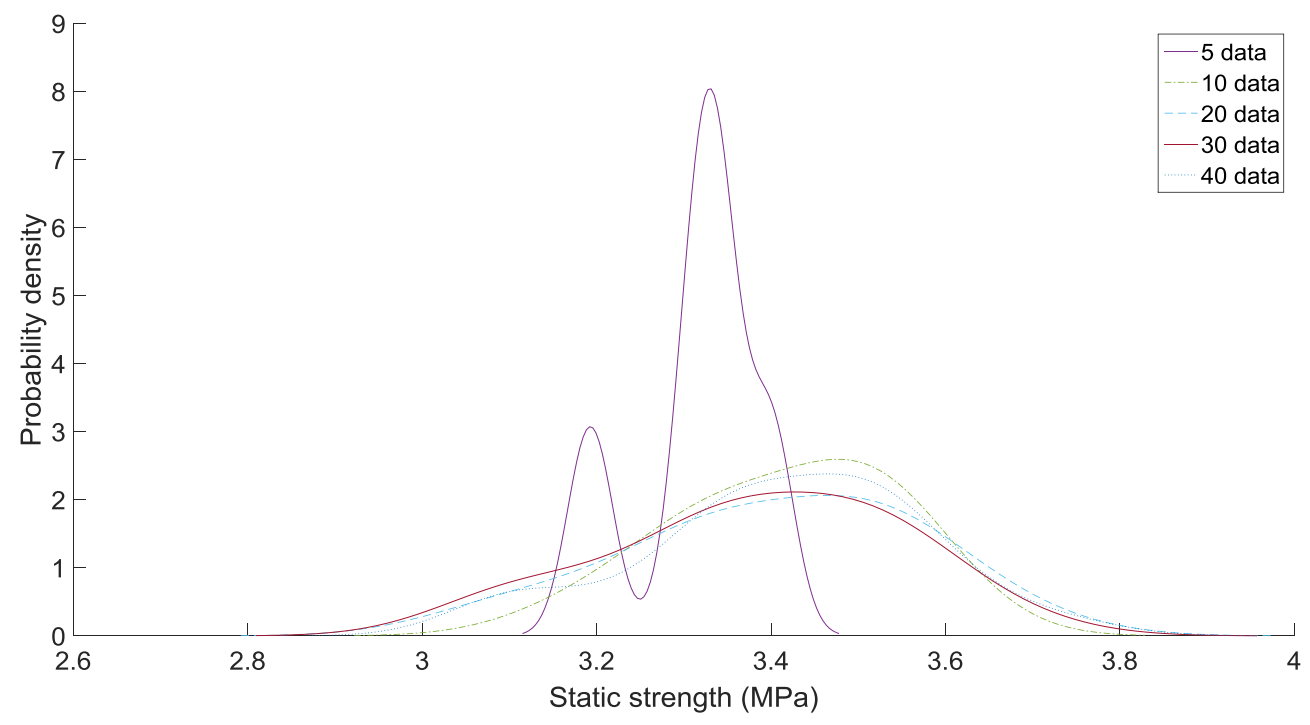

1) SFRC with $1 \%$ spiral fibres

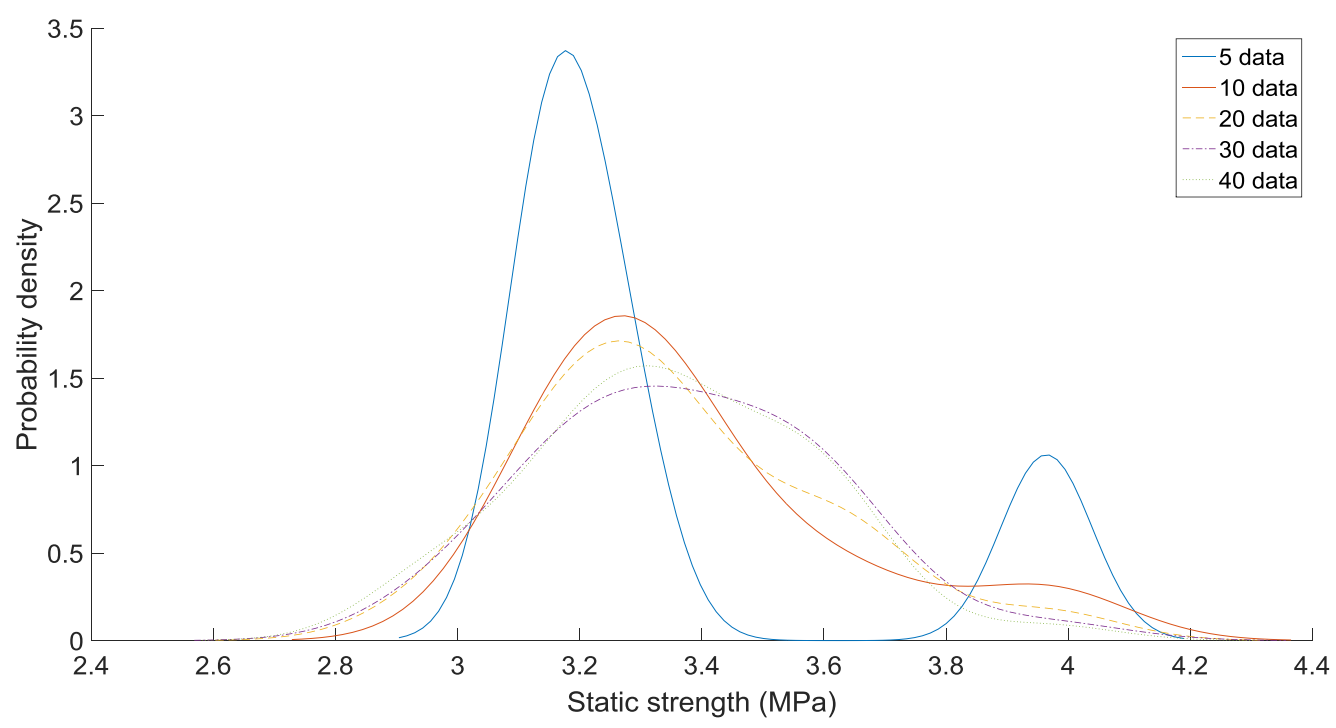

2) SFRC with $2 \%$ spiral fibres 


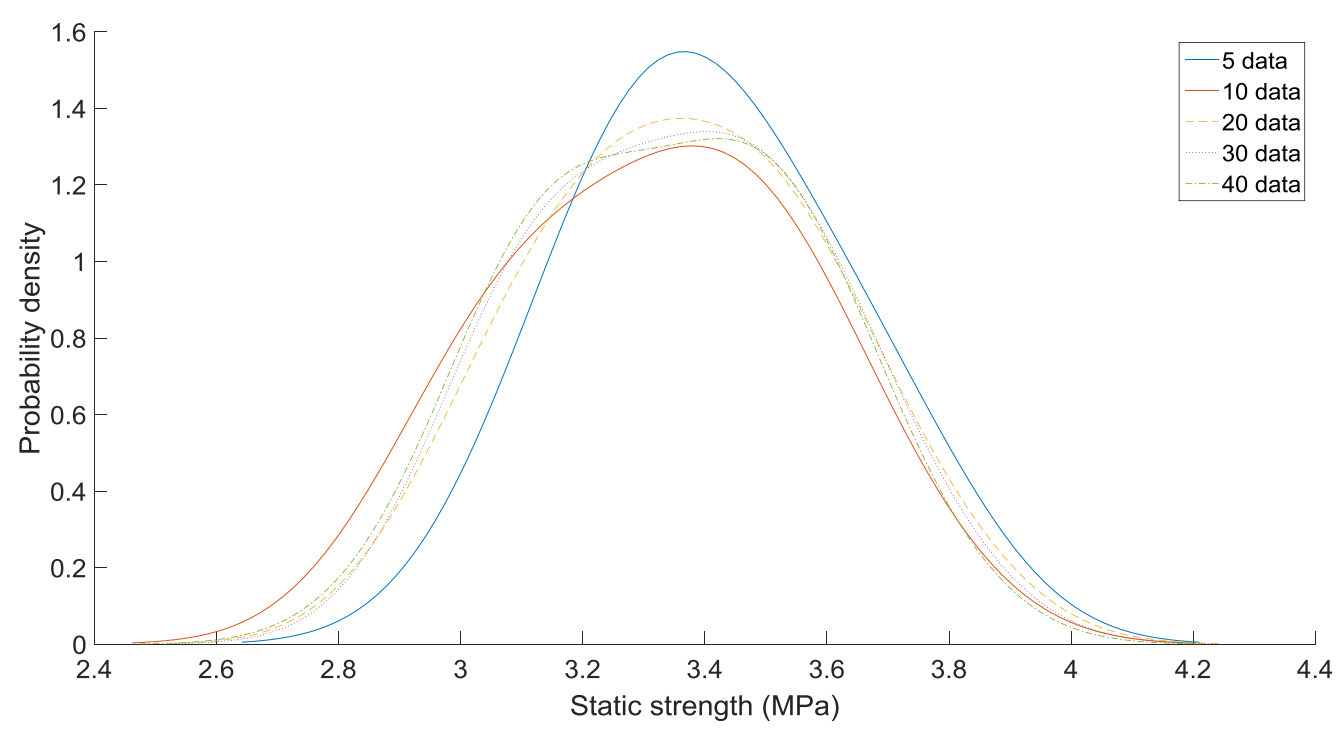

3) SFRC with 3\% spiral fibres

Figure 8 . Kernel density estimation using limited number of data

\section{Prediction model for the DIF of SFRC}

The 1D regression models were firstly constructed using the 1D form of Eq. (3), which is the relationship between DIF for tensile strength $(\mathrm{Y})$ and the strain rate $(\mathrm{X})$, under a certain concrete strength and a certain steel fibre ratio. The sampled data $\left(\mathrm{X}_{i}, \mathrm{Y}_{i}\right)$ were inputted into Eq. (3), and the equation became the regression model. This model can then be used to predict the new DIF values based on strain rate values. The detailed procedures for kernel selection and parameter determination can be found in Wang et al. [18].

Fig. 9 shows the kernel regression results between DIF for tensile strength and the strain rate, with different fibre ratios and static split tensile strengths. Specifically, for concrete with a certain ratio of steel fibres $(1 \%, 2 \%$, and 3\%), 10 kernel regression curves were obtained for 10 samples with different strengths, each with 17 different strain rates and DIF values. In each figure, the red line represents the fitted kernel regression results. The dashed blue lines have the same shape as the regression curve, but have a \pm 0.2 gap, which represent the tolerable error. Since the DIF values are normally higher than 2 , the tolerable error of 0.2 was adopted to make sure that the maximum difference between the predicted values and numerical results is less than $10 \%$. The numerical results are shown as the blue "+", with a red "o" when the real results are not within the tolerable lines for comparison. Specifically, the results with a red "o" are different from the fitted results, and the differences between them are larger than 0.2. As can be found from Fig. 9, all the samples fit well with the numerical simulation results for SFRC with $1 \%, 2 \%$, and $3 \%$ steel fibres, within the tolerable error. So no data was labelled as red "o". These results demonstrate the effectiveness of the kernel regression method. In contrast, by using experimental test results in Wang et al. [18], the data showed that a few "abnormal" results are not fitted well. The reason is that the numerical simulation is more consistent. Although randomness of the locations and directions of the fibres and aggregates are introduced to the numerical model, it still fluctuates less than the real concrete samples because other possible variations such as variation in material properties, construction quality, curing environments, etc are not considered in numerical model. To further demonstrate the effectiveness of the proposed regression method, the coefficient of determination $\left(R^{2}\right.$ value) for the regression models is calculated. The average $R^{2}$ values are $0.9990,0.9991$, and 0.9990 for SFRC with $1 \%, 2 \%$, and $3 \%$ fibres, respectively. 


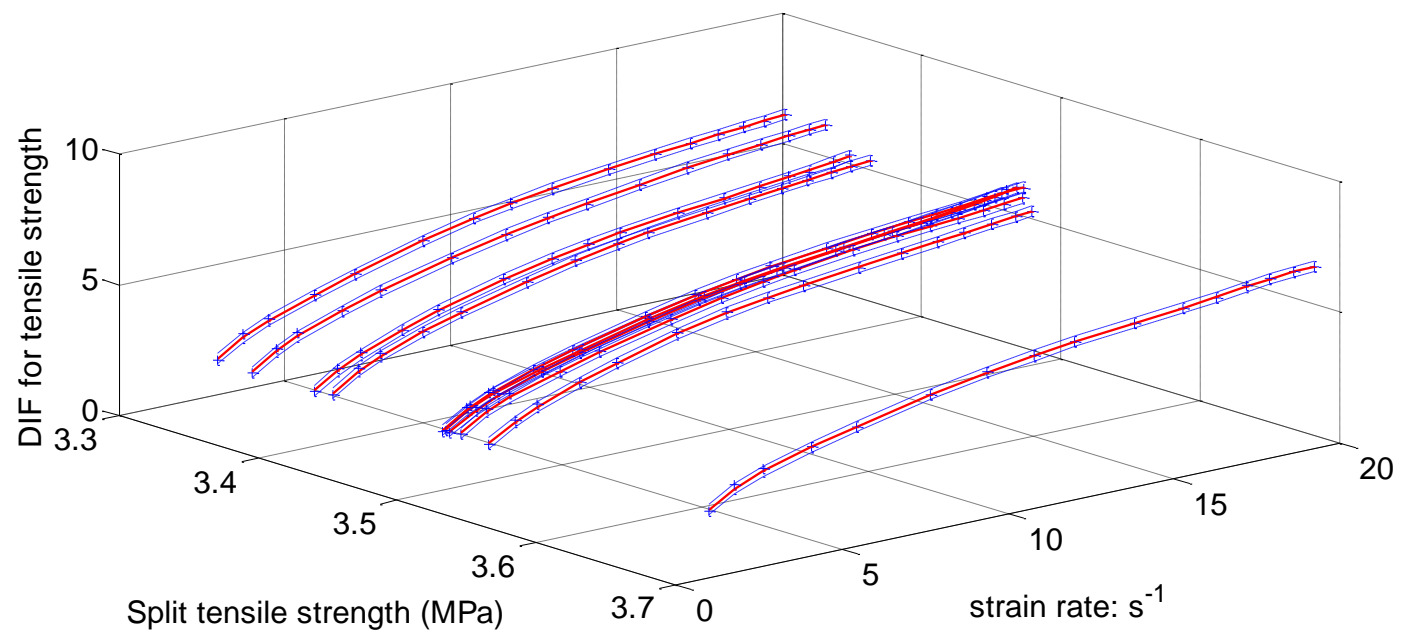

a) SFRC with $1 \%$ steel fibre

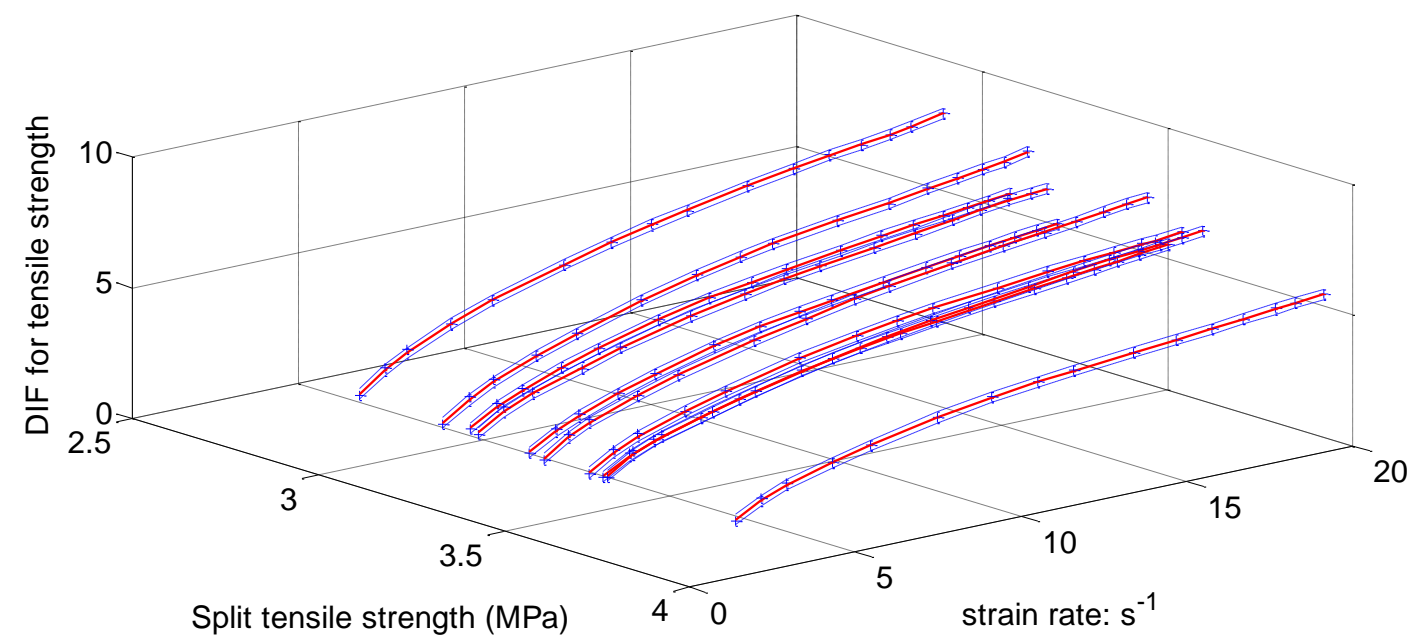

b) SFRC with $2 \%$ steel fibre

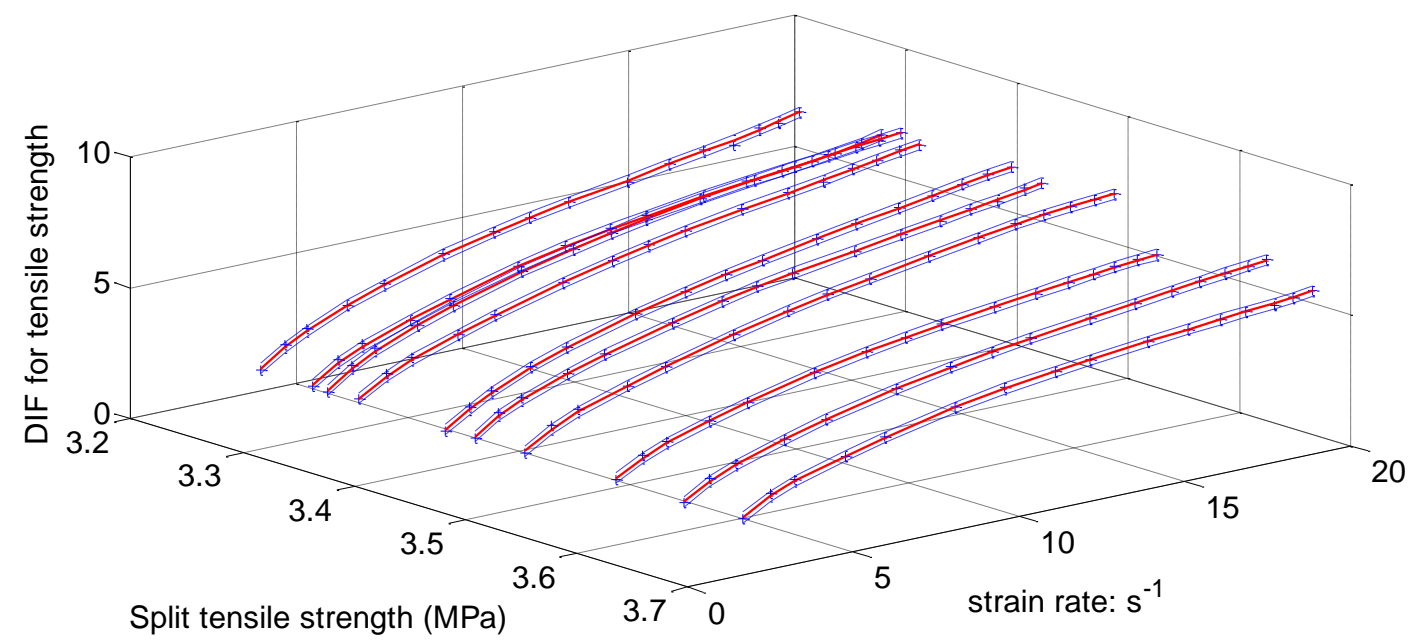

c) SFRC with $3 \%$ steel fibre

Figure 9. Regression model of DIF for tensile strength vs strain rate under different static strengths

It should be noted that the results presented in Fig. 9 are useful to identify the relationship between DIF and strain rate with specific fibre ratio and tensile strength. However, the concrete tensile strengths are 
usually random, because of the random nature of the material. Thus, the concrete strength should be considered as another variable to be incorporated in the prediction model of SFRC. Based on the steps 2-4 in Methodology presented above, the following results can be obtained. In this section, the increment for strain rate is taken as $0.5 \mathrm{~s}^{-1}$ and in the range of $1.2 \mathrm{~s}^{-1}$ to $20 \mathrm{~s}^{-1}$, and that for concrete tensile strength is $0.1 \mathrm{MPa}$, and covers from 2.5 MPa to 4.0 MPa. These incremental steps achieve the balance between a smooth surface shape and less data points.

Fig. 10 shows the relationship of DIF for tensile strength with respect to the strain rate and the static split tensile strength, which is a 2D model. On each surface, if the static split tensile strength of SFRC is known, its DIF at different strain rate can be determined. It can be seen that the fitted surfaces are smooth.

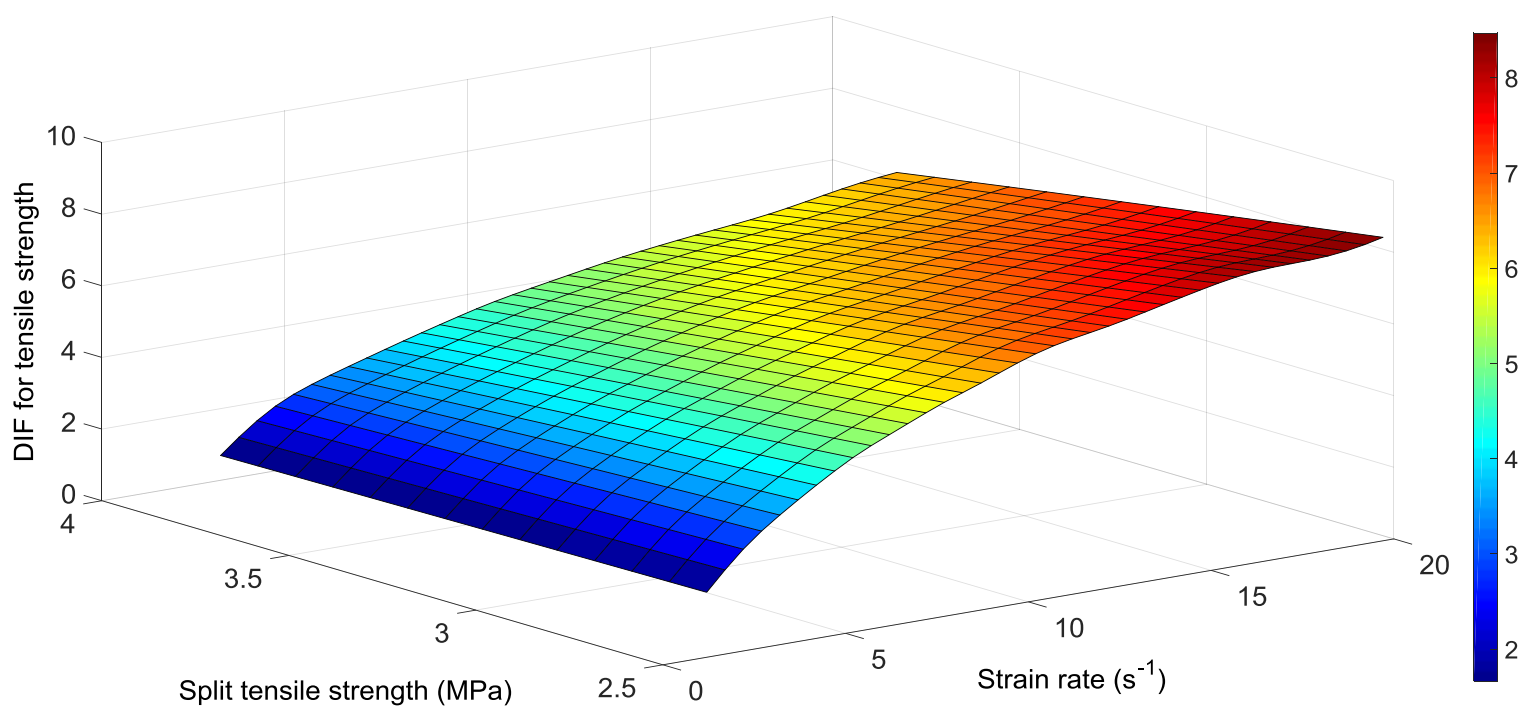

a) SFRC with $1 \%$ steel fibre

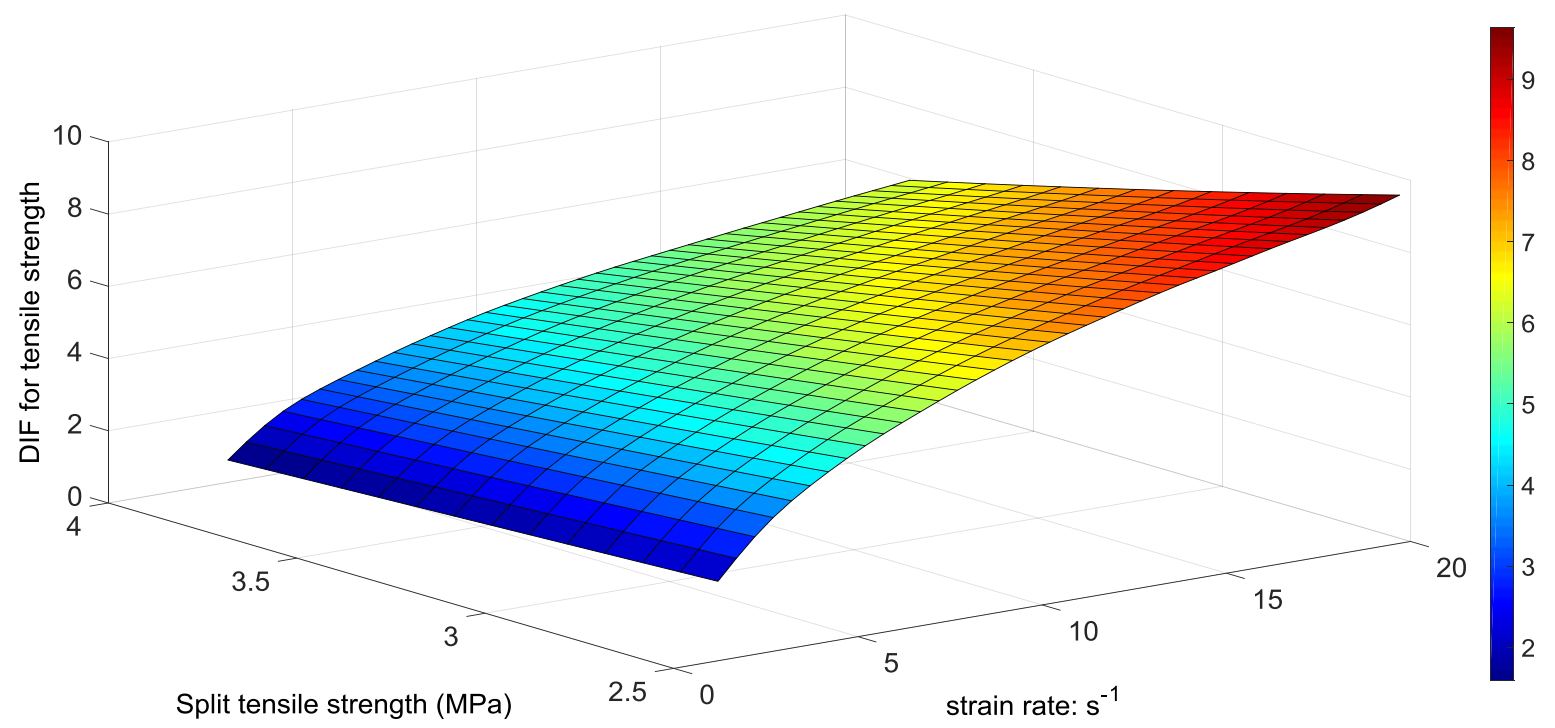

b) SFRC with $2 \%$ steel fibre 


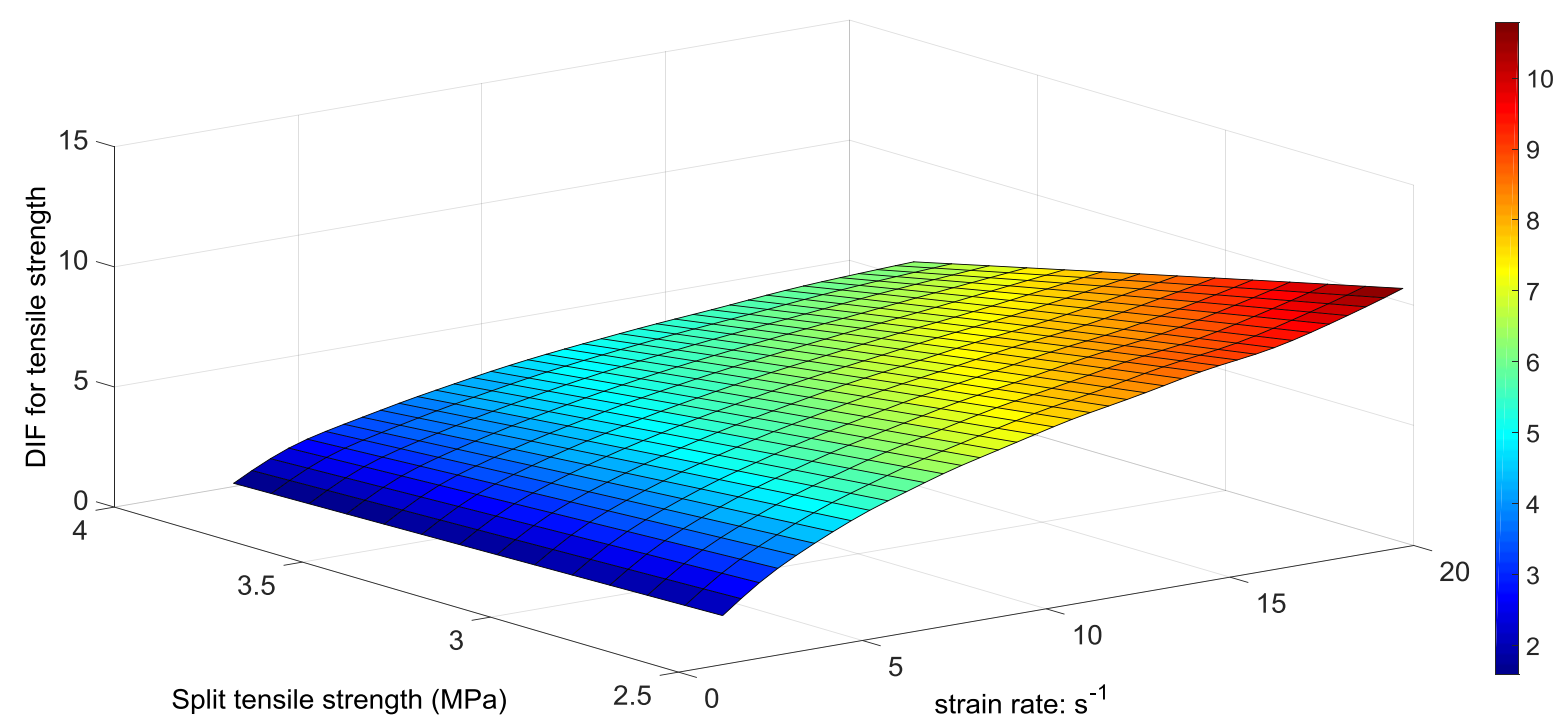

c) SFRC with $3 \%$ steel fibre

Figure 10. Prediction model of DIF for tensile strength vs strain rate and static tensile strength

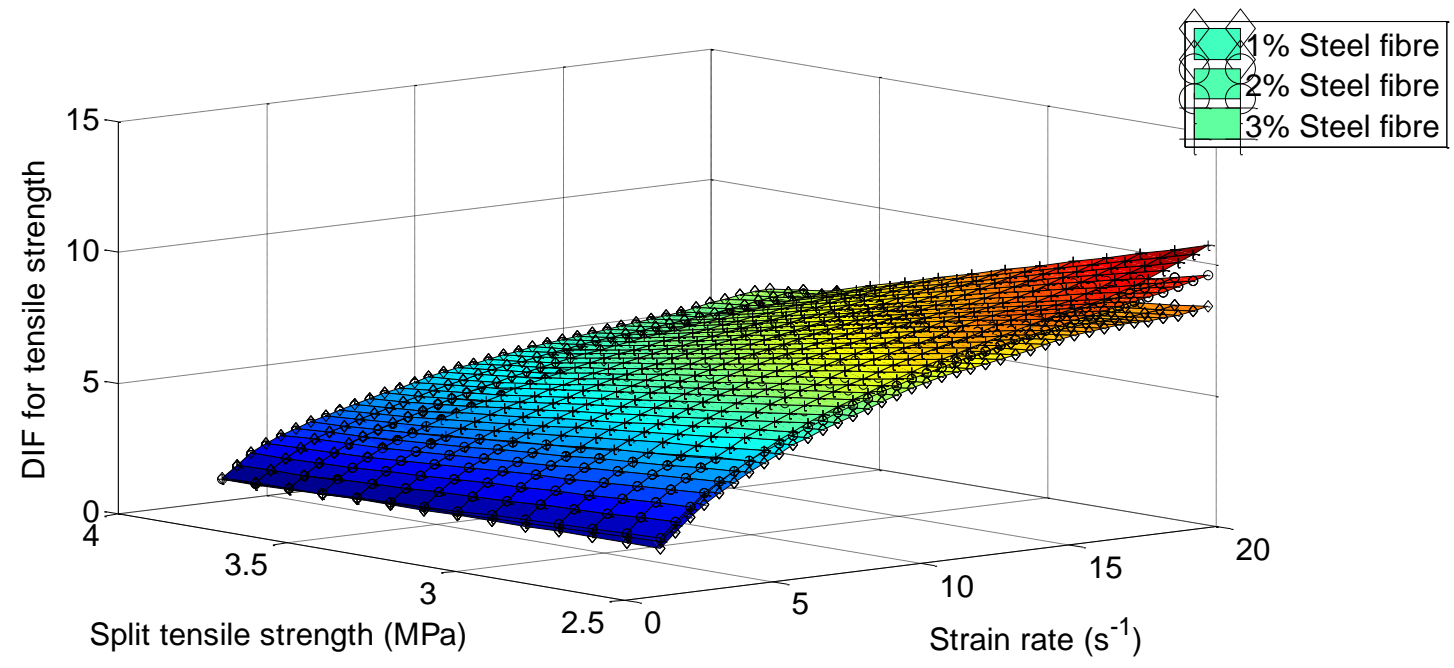

Figure 11. Comparison result for DIF vs strain rate and tensile strength

As shown in the figure, when strain rate is at a low level, the DIF value is less sensitive to the SFRC static tensile strength. When the strain rate is at a high level, the DIF values depend on the SFRC static tensile strength more significantly. For example, for the same concrete with $1 \%$ steel fibre, when the strain rate is $3.2 s^{-1}$, the DIF for SFRC with tensile strengths $3.0 \mathrm{MPa}$ and $3.6 \mathrm{MPa}$ are 3.386 and 3.11 , respectively. The difference is $8.15 \%$. When strain rate is $17.7 \mathrm{~s}^{-1}$, the DIF become 7.353 and 6.357 for 3.0MPa and 3.6MPa SFRC. The difference is $13.5 \%$. This effect becomes more significant when SFRC with more steel fibres is considered. Further, for SFRC with lower static tensile strengths, their DIF tends to be higher than those with higher tensile strengths at the same strain rate. One possible reason is the fibre distribution. In a SFRC sample, when more fibres are oriented to the same direction as that of the tensile force, the static strength should be higher. On the contrary, when more fibres are oriented randomly, the static strength is relatively lower, but the DIF is higher because the random distribution of fibres is able to provide more confinement to the concrete.

To investigate the effects of the ratio of the steel fibres, the three surfaces obtained were plotted together for comparison. As shown in Fig. 11, the three surfaces display similar trends. With the same split tensile strength and the same strain rate, the DIF for SFRC with higher ratio of steel fibres tends to be slightly higher indicating SFRC with more steel fibres is more strain rate sensitive. The difference is 
more significant when strain rate is higher and static tensile strength is lower. When strain rate is low and static strength is high, the DIF values for concrete with different ratios of steel fibres are very close to each other.

\section{Numerical verification of the proposed prediction model}

To verify the proposed prediction model for DIF of SFRC tensile strength, a new set of simulations were conducted for concrete with $1 \%, 2 \%$ and $3 \%$ steel fibre. As can be seen from Tables 5-7, the interpolated results are very close to the numerical simulation results. For concrete with $1 \%, 2 \%$ and $3 \%$ steel fibres, the average differences are $2.65 \%, 2.63 \%$, and $5.63 \%$, respectively. The static tensile strengths for concrete with $1 \%, 2 \%$ and $3 \%$ are $3.4423,3.46$, and $3.5676 \mathrm{MPa}$, respectively. Compared with the average strengths from the simulation results $(3.4876,3.4352$, and $3.4566 \mathrm{MPa}$ for concrete with $1 \%, 2 \%$, and $3 \%$ fibres, respectively), the differences are smaller when the strengths of the new set are closer to the average strengths (i.e., for concrete with $1 \%$ and $2 \%$ fibres), because more simulation data are available for these strengths in the prediction model. For concrete with strength not close to the averaged result, the prediction accuracy declines due to less data available. Adding more simulation data can solve this issue, which is not conducted in this study. These results demonstrate that the model developed from 10 data points only is sufficiently accurate, indicating the superiority of the proposed method in deriving the predictive models based on the limited number of data.

It should be noted that the proposed kernel regression method is flexible to different scenarios and thus it is able to find applications to the derivation of other statistical material properties and for different random materials, overcoming the difficulty of lack of data by using traditional methods.

Table 5: Comparison of DIF results for concrete with $1 \%$ steel fibre

\begin{tabular}{|c|c|c|c|c|}
\hline Load steps & Strain rate (1/s) & DIF from simulation & DIF from kernel regression & Difference \\
\hline Load_01 & 1.71 & 2.158 & 2.163 & $0.25 \%$ \\
\hline Load_02 & 2.52 & 2.922 & 2.792 & $-4.45 \%$ \\
\hline Load_03 & 3.20 & 3.265 & 3.183 & $-2.51 \%$ \\
\hline Load_04 & 4.49 & 3.750 & 3.742 & $-0.20 \%$ \\
\hline Load_05 & 5.62 & 4.239 & 4.162 & $-1.82 \%$ \\
\hline Load_06 & 7.43 & 4.774 & 4.752 & $-0.46 \%$ \\
\hline Load_07 & 8.99 & 5.134 & 5.186 & $1.01 \%$ \\
\hline Load_08 & 10.17 & 5.372 & 5.464 & $1.72 \%$ \\
\hline Load_09 & 11.13 & 5.541 & 5.652 & $2.01 \%$ \\
\hline Load_10 & 12.76 & 5.768 & 5.922 & $2.67 \%$ \\
\hline Load_11 & 13.99 & 5.913 & 6.113 & $3.37 \%$ \\
\hline Load_12 & 14.94 & 6.028 & 6.248 & $3.65 \%$ \\
\hline Load_13 & 15.75 & 6.143 & 6.355 & $3.45 \%$ \\
\hline Load_14 & 16.39 & 6.183 & 6.439 & $4.14 \%$ \\
\hline Load_15 & 17.03 & 6.196 & 6.526 & $5.32 \%$ \\
\hline Load_16 & 17.56 & 6.266 & 6.601 & $5.34 \%$ \\
\hline
\end{tabular}

Table 6: Comparison of DIF results for concrete with 2\% steel fibre

\begin{tabular}{|c|c|c|c|c|}
\hline Load steps & Strain rate (1/s) & DIF from simulation & DIF from kernel regression & Difference \\
\hline Load_01 & 1.72 & 2.135 & 2.209 & $3.45 \%$ \\
\hline Load_02 & 2.44 & 2.863 & 2.766 & $-3.39 \%$ \\
\hline Load_03 & 3.15 & 3.284 & 3.184 & $-3.04 \%$ \\
\hline Load_04 & 4.58 & 3.834 & 3.793 & $-1.07 \%$ \\
\hline
\end{tabular}




\begin{tabular}{|l|c|c|c|c|}
\hline Load_05 & 5.82 & 4.334 & 4.247 & $-2.00 \%$ \\
\hline Load_06 & 8.21 & 5.040 & 4.976 & $-1.27 \%$ \\
\hline Load_07 & 9.70 & 5.483 & 5.333 & $-2.73 \%$ \\
\hline Load_08 & 11.02 & 5.760 & 5.608 & $-2.65 \%$ \\
\hline Load_09 & 12.08 & 5.992 & 5.802 & $-3.16 \%$ \\
\hline Load_10 & 13.97 & 6.316 & 6.11 & $-3.27 \%$ \\
\hline Load_11 & 15.44 & 6.563 & 6.339 & $-3.41 \%$ \\
\hline Load_12 & 16.80 & 6.742 & 6.544 & $-2.94 \%$ \\
\hline Load_13 & 17.69 & 6.867 & 6.671 & $-2.85 \%$ \\
\hline Load_14 & 18.45 & 6.956 & 6.779 & $-2.55 \%$ \\
\hline Load_15 & 19.19 & 7.075 & 6.887 & $-2.65 \%$ \\
\hline Load_16 & 19.76 & 7.096 & 6.976 & $-1.69 \%$ \\
\hline
\end{tabular}

Table 7: Comparison of DIF results for concrete with 3\% steel fibre

\begin{tabular}{|c|c|c|c|c|}
\hline Load steps & Strain rate (1/s) & DIF from simulation & DIF from kernel regression & Difference \\
\hline Load_01 & 1.77 & 2.205 & 2.153 & $-2.38 \%$ \\
\hline Load_02 & 2.55 & 3.006 & 2.731 & $-9.15 \%$ \\
\hline Load_03 & 3.29 & 3.405 & 3.138 & $-7.84 \%$ \\
\hline Load_04 & 4.71 & 3.941 & 3.712 & $-5.81 \%$ \\
\hline Load_05 & 5.83 & 4.384 & 4.113 & $-6.19 \%$ \\
\hline Load_06 & 7.83 & 5.034 & 4.722 & $-6.19 \%$ \\
\hline Load_07 & 9.47 & 5.448 & 5.134 & $-5.77 \%$ \\
\hline Load_08 & 10.72 & 5.724 & 5.399 & $-5.68 \%$ \\
\hline Load_09 & 11.83 & 5.970 & 5.607 & $-6.08 \%$ \\
\hline Load_10 & 13.62 & 6.275 & 5.908 & $-5.86 \%$ \\
\hline Load_11 & 15.00 & 6.477 & 6.126 & $-5.42 \%$ \\
\hline Load_12 & 16.09 & 6.653 & 6.299 & $-5.33 \%$ \\
\hline Load_13 & 16.93 & 6.794 & 6.426 & $-5.42 \%$ \\
\hline Load_14 & 17.68 & 6.840 & 6.531 & $-4.52 \%$ \\
\hline Load_15 & 18.32 & 6.925 & 6.618 & $-4.44 \%$ \\
\hline Load_16 & 18.91 & 6.978 & 6.696 & $-4.04 \%$ \\
\hline
\end{tabular}

\section{Statistical model for SFRC}

Based on the above prediction surfaces, the statistical model for SFRC with different percentage of steel fibres can be obtained. From the obtained data, the statistical model for SFRC with different fibre ratios can be obtained using kernel regression method, i.e., Eq. (4). The only difference is the definition of $\left(X_{i}, Y_{i}\right)$. Specifically, $X_{i}$ is the available data point in the predication model, and that $Y_{i}$ is the mean value at $X_{i}$, which is calculated from the prediction model.

The mean values and standard deviations are shown with symbols + in Fig. 12 and Fig. 13, respectively, together with their regression curves $(1,2,3$ denotes $1 \%, 2 \%$, and $3 \%$ SFRC). It should be noted that all the statistical models are obtained from a universal equation, Eq. (4). The equation is dependent on the data, so this method has the advantage of flexibility and the obtained models can achieve much better agreement with the data. This can be demonstrated that almost all the data points in the regression models are equal to those obtained from prediction models. 
The statistical DIF values for SFRC with higher percentage of steel fibres are slightly higher. This is more evident when the strain rate increases. However, the maximum difference is only 5.3\%, which occurs between SFRC with $1 \%$ and 3\% steel fibres (6.913 and 7.28, respectively), when strain rate is equal to $20 s^{-1}$.

As can be seen from Fig. 13, the standard deviation values increase with the increase of strain rate. When the strain rate is at $1.2 s^{-1}$, the standard deviations reach their lowest value, i.e., $0.0441,0.2048$, and 0.1308 for SFRC with $1 \%, 2 \%$, and $3 \%$ steel fibres, respectively. The maximum standard deviations are $0.6937,1.205$, and 1.4582 , respectively. Clearly, when strain rate is high, the structural response becomes more random.

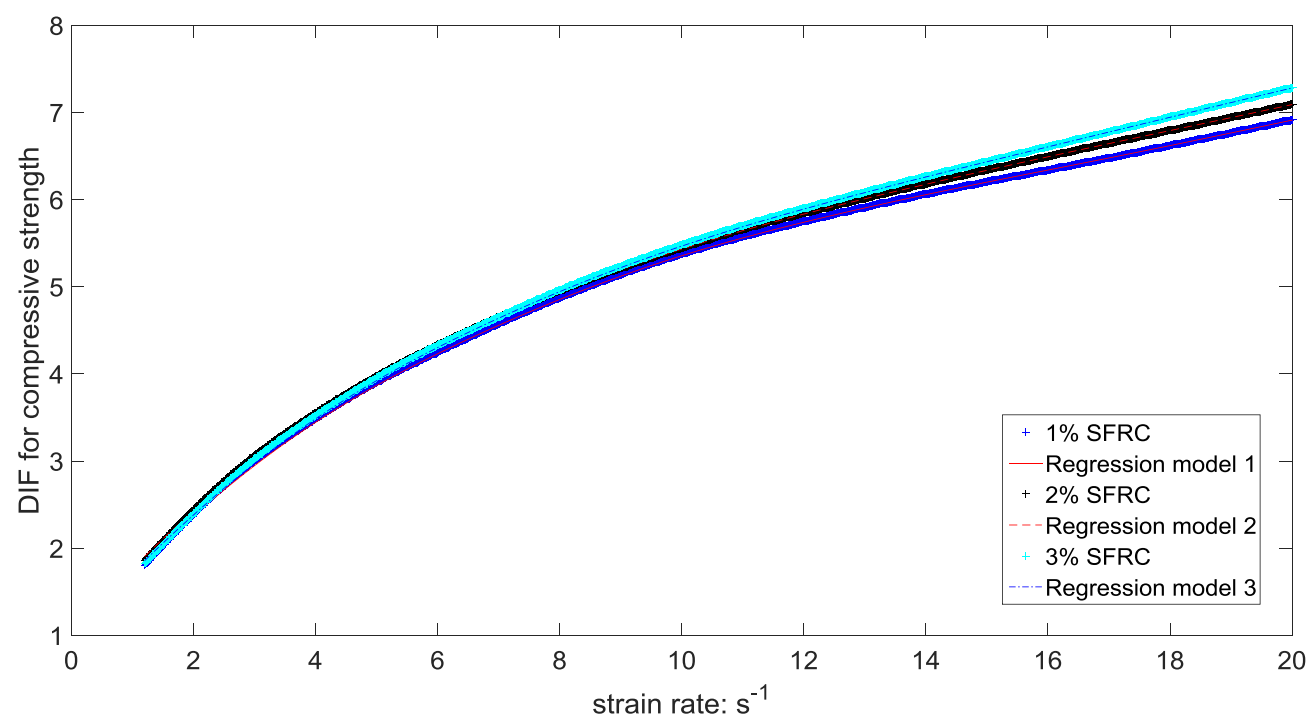

Figure 12. Statistical mean values for DIF of tensile strength vs strain rate

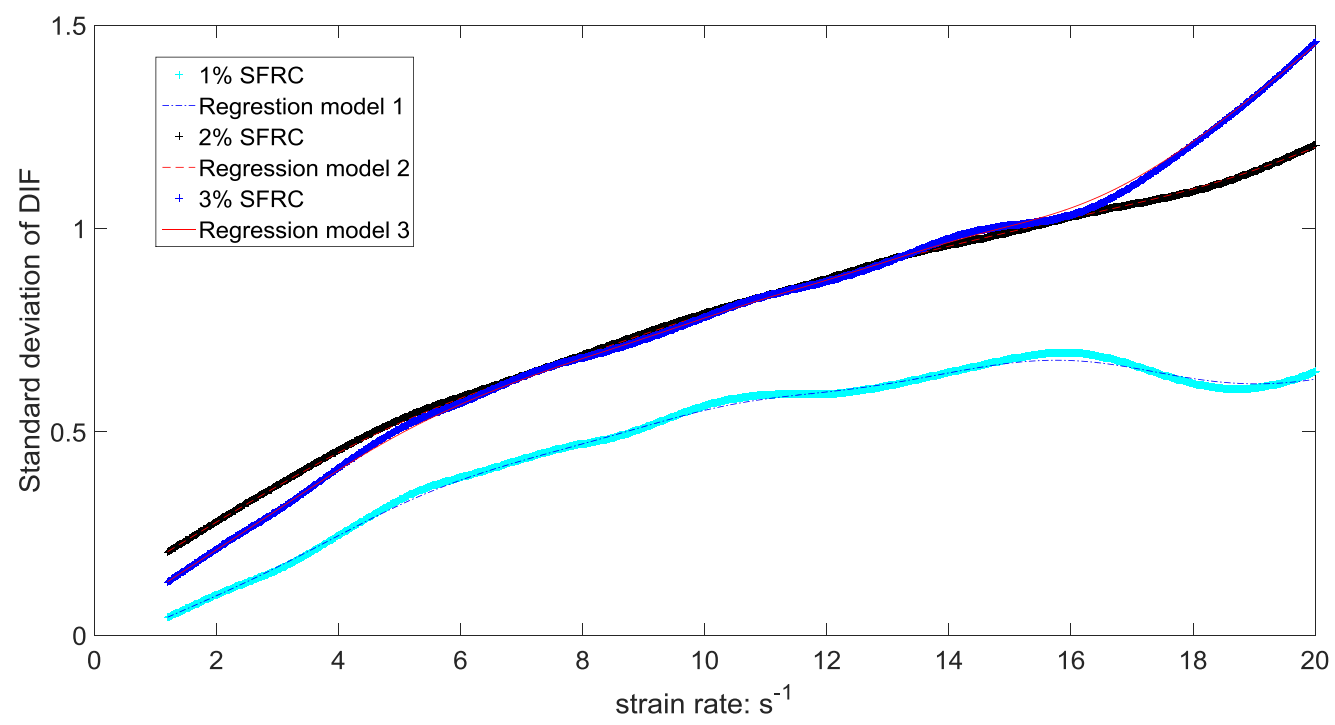

Figure 13. Standard deviation results for DIF of tensile strength vs strain rate

The distributions for SFRC at different strain rates can be obtained from the results of the prediction models. The DIF value at a certain strain rate and associated with a certain static strength can be determined from the above prediction model. Using the static strengths of 10 simulated samples, 10 DIF values at a certain strain rate can be obtained. By using KDE, the distribution of the obtained DIF values can be estimated. Typical distributions for SFRC with 1\%, 2\%, and 3\% fibres at $10 \mathrm{~s}^{-1}$ strain rate is shown in Fig. 14, which is further compared with the estimated distributions of static strengths 
in Fig. 15. It can be seen that the DIF distribution is to a certain extent dependent on the distribution of static strength. Specifically, the variances of $1 \%$ SFRC are the smallest while those of $2 \%$ SFRC are the largest. The results demonstrate that the proposed prediction model is suitable for finding the distribution of DIF values at different strain rates.

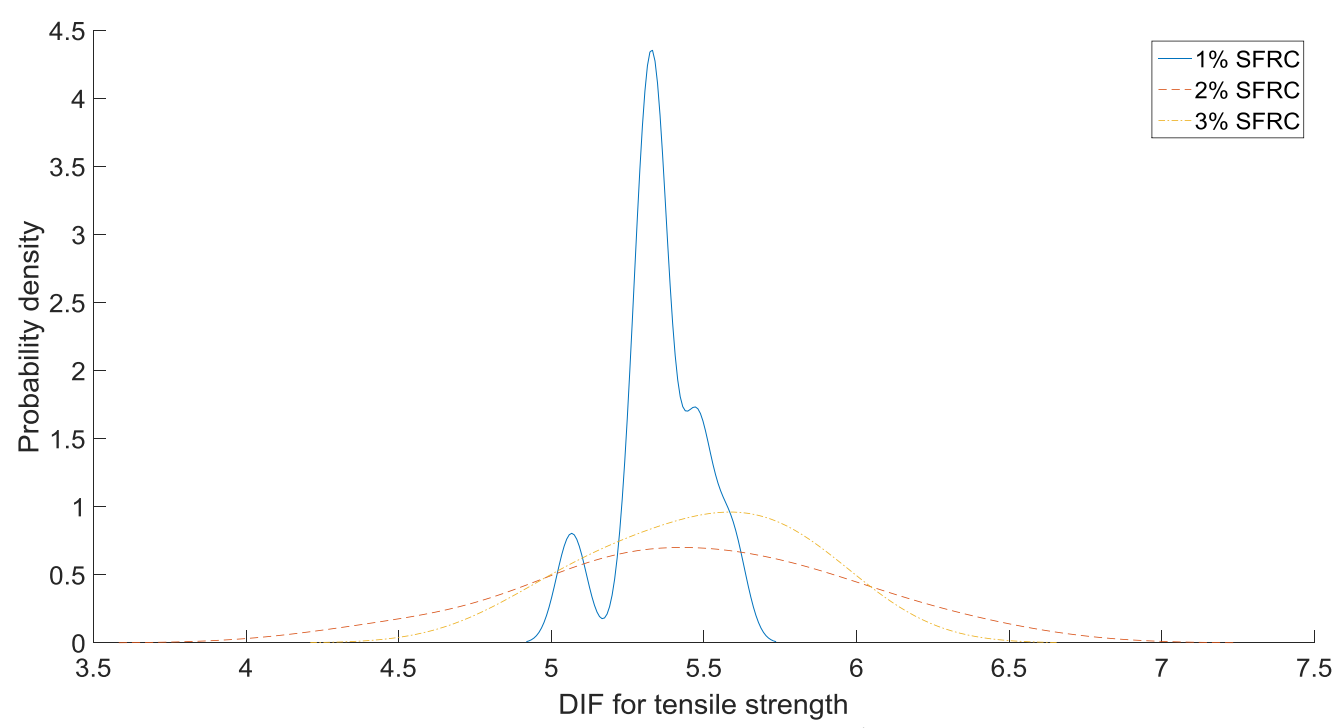

Figure 14. DIF distribution at $10 \mathrm{~s}^{-1}$ strain rate

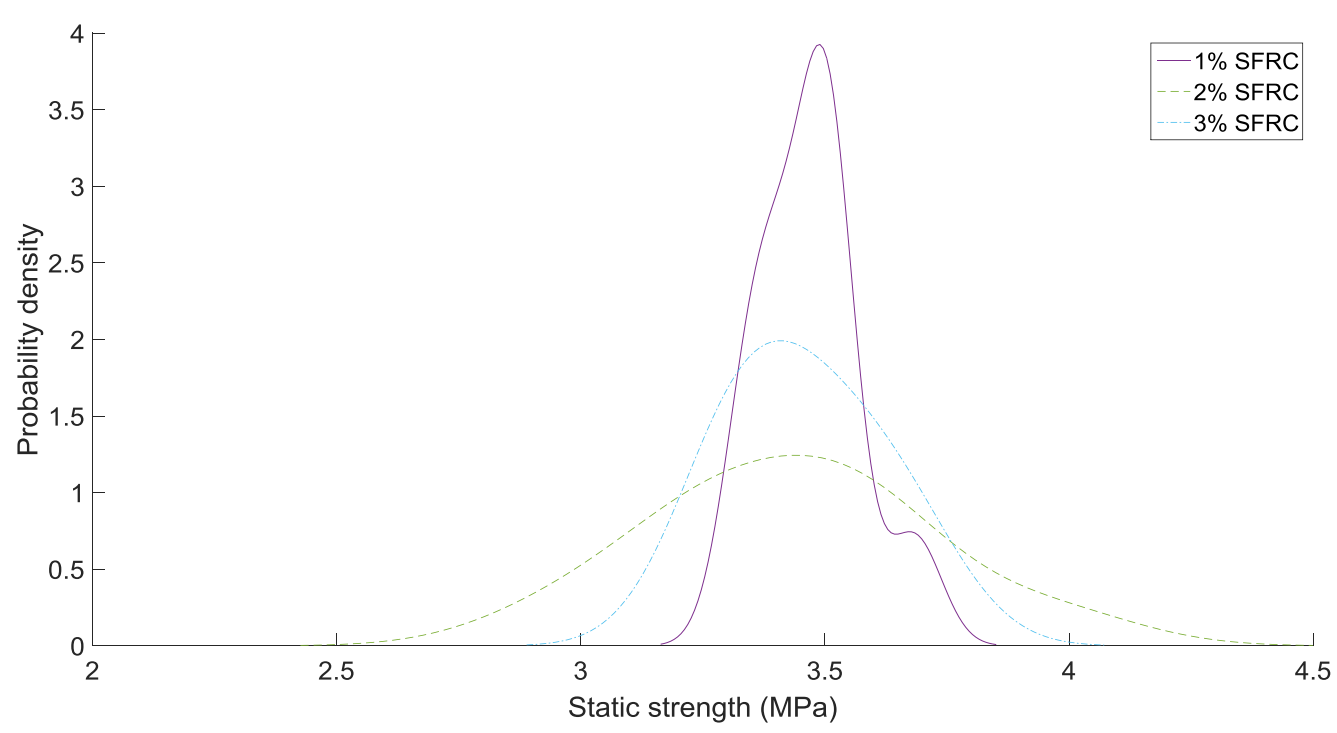

Figure 15. Estimated distributions of static strengths

\section{CONCLUSIONS}

This paper proposes a new method for the modelling of material properties of SFRC with different percentages of randomly distributed steel fibres. Instead of performing massive Monte Carlo simulation, nonparametric statistical methods, i.e., KDE and kernel regression method, are used. With a limited number of samples, the proposed method is able to find optimal regression surface and to achieve good prediction of the material properties. The numerical verification results confirm its applicability. The proposed method can be applied to estimating the statistical properties of any other materials with limited number of data, overcoming the requirements of extensive laboratory testing and/or numerical simulations. 


\section{ACKNOWLEDGMENTS}

The authors gratefully acknowledge the financial support provided by the Australian Research Council.

\section{REFERENCES}

1. Shah SP. (1991) "Do fibres increase the tensile strength of cement-based matrix?" ACI Material Journal, Vol. 88, No. 6, pp. 595-602.

2. Mindess S. and Zhang L. (2009) "Impact resistance of fibre-reinforced concrete" Proceedings of the ICE - Structures and Buildings, Vol. 162, No. 1, pp. 69-76

3. Brandt A.M., (2008) "Fibre reinforced cement-based (FRC) composites after over 40 years of development in building and civil engineering" Composite Structures, Vol. 86, No. 1-3, pp. 3-9

4. Xu Z., Hao. H., and Li H.N. (2012a) "Experimental study of dynamic compressive properties of fibre reinforced concrete material with different fibres", Materials \& Design, Vol. 33, pp. 42-55.

5. Xu Z., Hao. H., and Li H.N. (2012b) "Dynamic tensile behaviour of fibre reinforced concrete with spiral fibres", Materials \& Design, Vol. 42, pp. 72-88.

6. Xu Z., Hao. H., and Li H.N. (2012c) "Mesoscale modelling of dynamic tensile behaviour of fibre reinforced concrete with spiral fibres", Cement and Concrete Research, Vol. 42, No. 11, pp. 1475-1493.

7. Xu Z., Hao. H., and Li H.N. (2012d) "Mesoscale modelling of fibre reinforced concrete material under compressive impact loading", Construction and Building Materials, Vol. 26, No. 1, pp. 274-288.

8. Hao Y. and Hao H. (2013a) "Dynamic compressive behaviour of spiral steel fibre reinforced concrete in split Hopkinson pressure bar tests", Construction and Building Materials, Vol. 48, pp. $521-532$

9. Hao Y. and Hao H. (2016). "Mechanical properties and behaviour of concrete reinforced with spiral-shaped steel fibres under dynamic splitting tension". Magazine of Concrete Research, published online. DOI: 10.1680/jmacr.15.00372.

10. Hao Y. and Hao H. (2015). "Performance of spiral-shaped steel fibre reinforced concrete structure under static and dynamic loads", $2^{\text {nd }}$ International Conference on Performance-based and Life-cycle Structural Engineering, 9-11 December 2015, Brisbane, Australia

11. Song PS, Wu JC, Hwang S and Sheu BC., (2005) "Statistical analysis of impact strength and strength reliability of steel-polypropylene hybrid fiber-reinforced concrete', Construction and Building Materials, Vol.19, pp. 1-9.

12. Kang S.T., Lee B.Y., Kim J.K. and Kim Y.Y. (2011) "The effect of fibre distribution characteristics on the flexural strength of steel fibre-reinforced ultra high strength concrete", Construction and Building Materials, Vol. 25, No. 5, pp. 2450-2457

13. Cunha V.M.C.F., Barros J.A.O. and Sena-Cruz J.M. (2011) "An integrated approach for modelling the tensile behaviour of steel fibre reinforced self-compacting concrete" Cement and Concrete Research, Vol. 41, pp. 64-76.

14. Fang Q. and Zhang J. (2012). "Three-dimensional numerical modelling of concrete-like materials subjected to dynamic loadings". Advances in Protective Structures Research, Vol. 1, pp. 33-64.

15. Xu T., Zhao G., Zhu W., Chen C., and Yuan L. (2015) "Mesoscale modelling of spallation failure in fiber-reinforced concrete slab due to impact loading", International Journal of Geomechanics, B4015001.

16. Fang Q. and Zhang J. (2013). "Three-dimensional modelling of steel fiber reinforced concrete material under intense dynamic loading". Construction and Building Materials, Vol. 44, pp. 118-132.

17. Basu B., Tiwari D., Kundu D., and Prasad R. (2009) "Is Weibull distribution the most appropriate statistical strength distribution for brittle materials?" Ceramics International, Vol. 35, pp. 237-246

18. Wang Y., Hao H. and Hao Y.F. (2015) "Derivation of dynamic material properties of steel-fibre-reinforced concrete using kernel regression." $3^{\text {rd }}$ International Conference on Protective Structures. 3-6 February 2015, Newcastle, Australia

19. Silverman B.W. (1986) Density Estimation for Statistics and Data Analysis. CRC press. 
20. Tedesco J.W., Ross C.A. and Kuennen S.T. (1993). "Experimental and numerical analysis of high strain rate splitting tensile tests". ACI Materials Journal, Vol. 90, No. 2, pp. 162-169.

21. Walraven J.C. (1981). "Theory and experiments on the mechanical behaviour of cracks in plain and reinforced concrete subjected to shear loading", Heron 26.

22. Chen, G., Hao, Y. and Hao, H. (2015). "3D meso-scale modelling of concrete material in spall tests". Materials and Structures, Vol. 48, pp. 1887-1899.

23. Hao Y. and Hao H. (2011). "Numerical evaluation of the influence of aggregates on concrete compressive strength at high strain rate". International Journal of Protective Structures, Vol. 2, No. 2, pp. 177-206.

24. Hao Y. and Hao H. (2014). "Influence of the concrete DIF model on the numerical predictions of RC wall responses to blast loadings". Engineering Structures, Vol. 73, pp. 24-38.

25. Hao Y. and Hao H. (2013b). "Numerical investigation of the dynamic compressive behaviour of rock materials at high strain rate". Rock Mechanics and Rock Engineering, Vol. 46, pp. 373-388.

26. Hao Y., Hao H. Jiang G.P., and Zhou Y. (2013) "Experimental confirmation of some factors influencing dynamic concrete compressive strengths in high-speed impact tests", Cement and Concrete Research, Vol. 52, pp. 63-70. 